\title{
Sums of distinct squares
}

\author{
by
}

Paul T. Bateman (Urbana, Ill.), Adolf J. Hildebrand (Urbana, Ill.) and George B. Purdy (Cincinnati, Ohio)

1. Introduction. Throughout this paper we shall suppose that $s$ is an integer $\geq 5$. Then order of magnitude considerations show that every sufficiently large integer is expressible as a sum of $s$ distinct non-zero squares. In fact, E. M. Wright [Wr] proved that, if $s \geq 5$, then for large $n$ we can essentially prescribe the ratios of the squares in expressing $n$ as a sum of $s$ squares. Thus, for each $s \geq 5$ there exists a largest integer $N(s)$ which is not expressible as a sum of $s$ distinct non-zero squares. In this paper we shall obtain asymptotic estimates for $N(s)$.

In a recent paper [HK], Halter-Koch considered representations of integers as sums of $s$ distinct non-zero coprime squares, and he proved among other things the following results.

TheOREM 0 (Halter-Koch). The largest odd integer not expressible as a sum of 4 distinct non-zero squares with greatest common divisor 1 is 157 . Moreover, if $N^{*}(s)$ denotes the largest integer not expressible as a sum of $s$ distinct non-zero squares with greatest common divisor 1 , then $N^{*}(5)=245$, $N^{*}(6)=333, N^{*}(7)=330, N^{*}(8)=462, N^{*}(9)=539, N^{*}(10)=647$, $N^{*}(11)=888$, and $N^{*}(12)=1036$.

Halter-Koch also proved a number of related results. For example, he showed that for $s \geq 5$,

$$
N^{*}(s+1) \leq 2\left(\sqrt{N^{*}(s)}+2\right)^{2},
$$

which enables one to derive an explicit (but rather crude) bound for $N^{*}(s)$.

Of the two quantities $N(s)$ and $N^{*}(s)$, the former is the more natural one, and we shall express our results in terms of $N(s)$. Trivially, we have $N^{*}(s) \geq N(s)$ for all $s \geq 5$, and we shall show in Theorem 5 that the two functions are in fact identical. Thus, the coprimality condition in the definition of $N^{*}(s)$ does not affect the results in any way.

Research of the second author supported in part by an NSF Grant. 
Since any sum of $s$ distinct positive squares must be greater than or equal to the sum of the first $s$ positive squares, namely

$$
P(s)=\sum_{i=1}^{s} i^{2}=s(s+1)(2 s+1) / 6,
$$

we have the trivial lower bound $N(s) \geq P(s)-1$. In fact, $N(s)$ must be strictly larger than $P(s)$ since, for example, $P(s)+1$ is not expressible as a sum of $s$ distinct squares. Our principal result (Theorem 1) shows among other things that $N(s)$ is asymptotically equal to this lower bound $P(s)$ and gives a fairly precise estimate for the difference

$$
R(s)=N(s)-P(s) .
$$

In order to state this main theorem, we define $\lambda_{s} \geq 0$ by

$$
\lambda_{s}^{2}=2 \max (\|\sqrt{2 s}\|,\|\sqrt{2 s}-1 / 2\|),
$$

where $\|\cdot\|$ denotes the distance to the nearest integer. It is easy to see that

$$
\lambda_{s}^{2}=1 / 2+\|\sqrt{8 s}-1 / 2\| .
$$

We further set, for any non-negative real number $x$,

$$
\mathcal{L}_{x}=\log \log \max \left(x, e^{e}\right), \quad t_{x}=\left\lfloor\mathcal{L}_{x} / \log 2\right\rfloor, \quad f(x)=\sum_{i=0}^{t_{x}} x^{2^{-i}} .
$$

THEOREM 1. (i) We have the asymptotic formula

$$
R(s)=2 s\left\{\sqrt{2 s}+\lambda_{s}(2 s)^{1 / 4}+O\left(s^{1 / 8}\right)\right\} .
$$

(ii) We have the upper estimate

$$
R(s) \leq 2 s\left\{f(\sqrt{2 s})+O\left(\mathcal{L}_{s}^{2}\right)\right\} .
$$

(iii) The bound (1.3) is best possible in the sense that there exists an increasing sequence $\left\{s_{k}\right\}$ of positive integers such that

$$
R\left(s_{k}\right) \geq 2 s_{k}\left\{f\left(\sqrt{2 s_{k}}\right)+O\left(\mathcal{L}_{s_{k}}\right)\right\} .
$$

An example of such a sequence is given by taking $s_{1}=1$ and $s_{k}=2 s_{k-1}^{2}+$ $s_{k-1}$ for $k \geq 2$.

The estimate $(1.2)$ shows in particular that $R(s) \ll \sqrt{P(s)}$. The second main term on the right-hand side of (1.2) involves the oscillatory quantity $\lambda_{s}$, which depends on how $8 s$ is situated relative to the sequence of squares. From the representation (1.1) of $\lambda_{s}$ it is clear that $1 / \sqrt{2} \leq \lambda_{s} \leq 1$ and that these bounds are best possible. Specifically, $\lambda_{s}$ will be near its maximal value 1 if $8 s$ is close to a square; and $\lambda_{s}$ will be near its minimal value $1 / \sqrt{2}$ when $8 s$ is roughly midway between two consecutive squares, for example, when $s$ has the form $m(8 m \pm 1)$. Thus, $R(s)=N(s)-P(s)$ oscillates between 
the limits $(2 s)^{3 / 2}+(2 s)^{5 / 4} / \sqrt{2}$ and $(2 s)^{3 / 2}+(2 s)^{5 / 4}$, up to an error term $O\left(s^{9 / 8}\right)$.

The inequality (1.3) gives a universal upper bound for $R(s)$ which sharpens that of (1.2) when $8 s$ is close to a square and which by (1.4) is best possible.

The remainder of this paper is organized as follows. In Section 2 we give an explicit polynomial upper bound for $N(s)$, namely $N(s)<(s-1)^{5}$ for $s \geq 5$ (Theorem 2), which will be needed as a basis for the subsequent arguments. In Section 3 we reformulate the problem of determining $N(s)$ and state a result (Theorem 3 ) about a related extremal problem. This problem concerns the minimum $Q(m)$ of $\sum_{i=1}^{t} a_{i}$ for all representations of the integer $m$ in the form $m=\sum_{i=1}^{t} \varepsilon_{i} a_{i}^{2}$, where $\varepsilon_{i}= \pm 1$ for all $i$ and $a_{1}, \ldots, a_{t}$ are distinct positive integers. Theorem 3 gives estimates for $Q(m)$ parallel to those of Theorem 1 and forms the principal ingredient in the proof of that theorem, but is also of some interest for its own sake. In Sections 4 and 5 we prove Theorem 3, and in Sections 6 and 7 we prove Theorem 1. In Section 8, we give the explicit upper bound (Theorem 4)

$$
N(s)<P(s)+2 s \sqrt{2 s}+44 s^{5 / 4}+108 s \quad(s \geq 166),
$$

which is useful for various purposes. In particular, we use (1.5) to show that the function $N(s)$ is monotonic for $s \geq 7$; this answers a question of Erdös. (Note, however, that the function $R(s)=N(s)-P(s)$ is not monotonic, since (1.2) gives $R\left(8 m^{2}\right)>R\left(8 m^{2}+m\right)$ for all large $m$.) In Section 9, we prove the above remark that $N(s)=N^{*}(s)$ for every $s \geq 5$; in fact, we show (Theorem 5) that if a positive integer is expressible as a sum of $s \geq 5$ distinct non-zero squares then it is also expressible as a sum of $s$ distinct non-zero squares with greatest common divisor 1 . In Section 10 we make some remarks on the more general problem of expressing an integer as a sum of $s$ distinct positive $k$ th powers. Using the results of Hardy and Littlewood on Waring's problem, we show (Theorem 6) that if $N_{k}(s)$ denotes the largest integer not expressible in this form, then

$$
N_{k}(s)=\frac{s^{k+1}}{k+1}+O\left(s^{k}\right) .
$$

In the final section, we discuss the computation of $N(s)$ and we give two tables of numerical data.

2. An initial upper bound. Using the result of Halter-Koch on four squares mentioned in the preceding section, we obtain the rough bound $N(s)<(s-1)^{5}$, which will be needed later on.

THEOREM 2. If $s \geq 5$ and if $n \geq(s-1)^{5}$, then $n$ is expressible as a sum of $s$ distinct non-zero squares. 
Proof. It is convenient to prove the assertion of the theorem under the slightly weaker assumption $n \geq(s-1)^{4}(s-3)$. For $i=1,2, \ldots, s-5$ we put $a_{i}=\lfloor\sqrt{n /(s-3)}\rfloor+i$; we also put $a_{s-4}=\lfloor\sqrt{n /(s-3)}\rfloor+s-4+\delta$, where $\delta \in\{0,1\}$ is chosen so that $r=n-a_{1}^{2}-a_{2}^{2}-\ldots-a_{s-4}^{2}$ is odd. (When $s=5$, only $a_{s-4}$ is needed.) Then $a_{i}^{2}>n /(s-3)$ for each $i$ and thus $r<n /(s-3)$. Moreover,

$$
r \geq n-\sum_{i=1}^{s-5}\left(\sqrt{\frac{n}{s-3}}+i\right)^{2}-\left(\sqrt{\frac{n}{s-3}}+s-3\right)^{2}=f_{s}(n),
$$

say. A simple calculation gives

$$
f_{s}(n)=\frac{n}{s-3}-\left(s^{2}-7 s+14\right) \sqrt{\frac{n}{s-3}}-\left(2 s^{3}-21 s^{2}+85 s-126\right) / 6 .
$$

Clearly $f_{s}(n)$ is an increasing function of $n$ provided $\sqrt{n /(s-3)}>\left(s^{2}-\right.$ $7 s+14) / 2$. This condition is satisfied if $s \geq 5$ and $n \geq(s-1)^{4}(s-3)$. Thus, if $n \geq(s-1)^{4}(s-3)$, we have

$$
r \geq f_{s}(n) \geq f_{s}\left((s-1)^{4}(s-3)\right)=\frac{14}{3} s^{3}-\frac{39}{2} s^{2}+\frac{101}{6} s+8 .
$$

The polynomial on the right-hand side here is an increasing function of $s$ for $s \geq 5$ and hence

$$
r \geq \frac{14}{3} 5^{3}-\frac{39}{2} 5^{2}+\frac{101}{6} 5+8=188 .
$$

Since $r$ is odd and greater than 157, Theorem 0 shows that $r$ is expressible as a sum of four distinct non-zero squares. Since each of these four squares is less than

$$
r<n /(s-3)<a_{1}^{2}<a_{2}^{2}<\ldots<a_{s-4}^{2}
$$

and since $n=r+a_{1}^{2}+a_{2}^{2}+\ldots+a_{s-4}^{2}$, the assertion of the theorem follows.

3. An extremal problem. In this section we rephrase the problem of estimating $N(s)$ in a form which is more suitable when dealing with integers that are close to $P(s)$, and we state a result (Theorem 3 ), which will form the principal ingredient in the proof of Theorem 1 . The underlying idea is that if $n$ is an integer close to $P(s)=\sum_{i=1}^{s} i^{2}$ which has a representation $n=\sum_{i=1}^{s} a_{i}^{2}$ as a sum of $s$ distinct squares, then the set $\left\{a_{i}: i \leq s\right\}$ can be expected to be "close" to the set $\{i: i \leq s\}$.

To make this idea precise, we note that any set $\left\{a_{i}: i \leq s\right\}$ of distinct positive integers can be obtained from the set $\{i: i \leq s\}$ by replacing some of the integers $i \leq s$, say $s-h_{i}, i \leq t$, by distinct integers $>s$, say $s+k_{i}$, 
$i \leq t$. The associated representation $n=\sum_{i=1}^{s} a_{i}^{2}$ can then be written as

$$
\begin{aligned}
n & =\sum_{i=1}^{s} i^{2}-\sum_{i=1}^{t}\left(s-h_{i}\right)^{2}+\sum_{i=1}^{t}\left(s+k_{i}\right)^{2} \\
& =P(s)+2 s \sum_{i=1}^{t}\left(h_{i}+k_{i}\right)+\sum_{i=1}^{t}\left(k_{i}^{2}-h_{i}^{2}\right),
\end{aligned}
$$

where the numbers $h_{i}$ and $k_{i}$ satisfy

$$
\begin{array}{ll}
h_{i} \text { distinct, } & 0 \leq h_{i}<s, \\
k_{i} \text { distinct, } & k_{i} \geq 1 .
\end{array}
$$

Conversely, any integer $n$ expressible in the form (3.1) with the conditions (3.2) and (3.3) is a sum of $s$ distinct positive squares. Therefore, $R(s)=$ $N(s)-P(s)$ is the largest integer $r$ not expressible in the form

$$
r=2 s \sum_{i=1}^{t}\left(h_{i}+k_{i}\right)+\sum_{i=1}^{t}\left(k_{i}^{2}-h_{i}^{2}\right)
$$

with integers $h_{i}$ and $k_{i}$ satisfying (3.2) and (3.3).

The above formulation leads naturally to the problem of minimizing the sum $\sum_{i=1}^{t}\left(h_{i}+k_{i}\right)$, subject to the conditions (3.2) and (3.3), while holding the sum $\sum_{i=1}^{t}\left(k_{i}^{2}-h_{i}^{2}\right)$ fixed. However, this extremal problem is somewhat awkward to deal with directly, as the conditions (3.2) and (3.3) are not symmetrical and depend on the parameter $s$. We therefore consider the following related, but simpler and more natural problem, which is sufficient for the application to the proof of Theorem 1 and also is of some intrinsic interest. For $m \neq 0$ set

$$
Q(m)=\min \left\{\sum_{i=1}^{t} a_{i}: \sum_{i=1}^{t} \varepsilon_{i} a_{i}^{2}=m\right\},
$$

where the minimum is taken over all sets $\left\{a_{i}: i \leq t\right\}$ of distinct positive integers satisfying $\sum_{i=1}^{t} \varepsilon_{i} a_{i}^{2}=m$ with suitable numbers $\varepsilon_{i} \in\{ \pm 1\}$, and define $Q(0)=0$. The quantity $Q(m)$ may be viewed as a measure for how "economically" $m$ can be represented as a difference of sums of distinct squares. The following result gives precise upper and lower bounds for $Q(m)$ that are largely parallel to those of Theorem 1 . Since $m=((m+10) / 2)^{2}-$ $((m+8) / 2)^{2}-3^{2}$ for even positive integers $m$ and $m=((m+17) / 2)^{2}-$ $((m+15) / 2)^{2}-4^{2}$ for odd positive integers $m$, every non-zero integer $m$ has indeed a representation $m=\sum_{i=1}^{t} \varepsilon_{i} a_{i}^{2}$ of the above form, so that $Q(m)$ is well-defined. Halter-Koch's result that $N^{*}(5)=245$, along with Schwarz's inequality, shows that trivially $Q(m) \leq \sqrt{5 m}$ for $m \geq 246$. 
THEOREM 3. (i) We have the asymptotic formula

$$
Q(m)=\sqrt{|m|}+\sqrt{2 \theta_{|m|}}|m|^{1 / 4}+O\left(|m|^{1 / 8}\right),
$$

where $\theta_{x}=\|\sqrt{x}\|$.

(ii) We have the upper estimate

$$
Q(m) \leq f(\sqrt{|m|})+O\left(\mathcal{L}_{|m|}\right)
$$

where $f(x)$ and $\mathcal{L}_{x}$ are defined as in Theorem 1.

(iii) The inequality (3.7) is best possible in the sense that if the sequence $\left\{m_{k}\right\}$ is defined by $m_{0}=1$ and $m_{k}=m_{k-1}^{2}+m_{k-1}$ for $k \geq 1$, then we have

$$
Q\left(m_{k}\right) \geq f\left(\sqrt{m_{k}}\right)+O\left(\mathcal{L}_{m_{k}}\right) .
$$

(iv) The upper bounds in (3.6) and (3.7) remain valid if in the definition (3.5) of $Q(m), t$ is restricted by the condition

$$
t \leq C \mathcal{L}_{|m|},
$$

where $C$ is a suitable absolute constant.

4. Proof of Theorem 3; upper bounds. Call a representation $m=$ $\sum_{i=1}^{t} \varepsilon_{i} a_{i}^{2}$ admissible if $\varepsilon_{i} \in\{ \pm 1\}$ and the numbers $a_{i}$ are distinct positive integers. To obtain the upper bounds of Theorem 3 (in the stronger form claimed in the last part of Theorem 3), we need to construct an admissible representation with $t \leq C \mathcal{L}_{|m|}$ for which the sum $\sum_{i=1}^{t} a_{i}$ is bounded by the right-hand sides of (3.6) and (3.7). Our construction is essentially that obtained by the greedy algorithm, supplemented by a direct argument for the first few values of $m$. We first dispose of the case of small $m$ with the following lemma.

LEMma 4.1. If $0<|m| \leq 37$, then $m$ has an admissible representation $m=\sum_{i=1}^{t} \varepsilon_{i} a_{i}^{2}$ such that $a_{i} \leq 5$ for all $i$.

Proof. The identities $1=1^{2}, 2=4^{2}-3^{2}-2^{2}-1^{2}, 3=2^{2}-1^{2}, 4=2^{2}$, $5=2^{2}+1^{2}, 6=3^{2}-2^{2}+1^{2}, 7=4^{2}-3^{2}, 8=3^{2}-1^{2}, 9=3^{2}, 10=3^{2}+1^{2}$, $11=4^{2}-2^{2}-1^{2}, 12=4^{2}-2^{2}$, and $13=3^{2}+2^{2}$ show that every $m$ with $0<m \leq 13$ has a representation of the required form with $a_{i} \leq 4$. Replacing $\varepsilon_{i}$ by $-\varepsilon_{i}$ in each of these representations, we see that the same is true for $-13 \leq m<0$. In the remaining range $13<|m| \leq 37$ the result follows by writing $m=\varepsilon 5^{2}+m^{\prime}$ with $\varepsilon \in\{ \pm 1\}$ and $\left|m^{\prime}\right| \leq 12$ and representing $m^{\prime}$ in the above form using squares $a_{i}^{2}$ with $a_{i} \leq 4$.

The lemma shows that for $0<|m| \leq 37, Q(m)$ is well-defined and satisfies the bounds (3.6) and (3.7) trivially, provided the $O$-constants are suitably chosen. The same is true for $m=0$, since by definition $Q(0)=0$. To deal with the general case, we begin with the following observation. Given an arbitrary integer $m$, let $q=\lfloor\sqrt{|m|}\rfloor$, so that $q^{2} \leq|m| \leq q^{2}+2 q$, and 
set $a=\langle\sqrt{|m|}\rangle$, where $\langle x\rangle$ denotes the nearest integer to $x$. (Note that, since $\sqrt{|m|}$ cannot be half an odd integer, there is no ambiguity in the definition of $\langle\sqrt{|m|}\rangle$.) Then $a=q$ if $q^{2} \leq|m| \leq q^{2}+q, a=q+1$ if $q^{2}+q+1 \leq|m| \leq q^{2}+2 q$, and in either case we have $m=\varepsilon a^{2}+r$ with $\varepsilon=\operatorname{sign}(m)$ (with the convention $\operatorname{sign}(0)=1)$ and $|r| \leq q=\lfloor\sqrt{|m|}\rfloor$.

Iterating this procedure, we obtain, for any given integer $m$, sequences of integers $\left\{a_{i}\right\}$ and $\left\{r_{i}\right\}$ defined by

$$
r_{0}=m, a_{i}=\left\langle\sqrt{\left|r_{i-1}\right|}\right\rangle, \varepsilon_{i}=\operatorname{sign}\left(r_{i-1}\right), r_{i-1}=\varepsilon_{i} a_{i}^{2}+r_{i}(i \geq 1),
$$

such that

$$
\left|r_{i}\right| \leq\left\lfloor\sqrt{\left|r_{i-1}\right|}\right\rfloor \quad(i \geq 1) .
$$

We then have for any $k \geq 1$ the representation

$$
m=\sum_{i=1}^{k} \varepsilon_{i} a_{i}^{2}+r_{k} .
$$

In fact, for sufficiently large $k$ we have the exact representation $m=$ $\sum_{i=1}^{k} \varepsilon_{i} a_{i}^{2}$, since it is easily seen that the sequence $\left\{r_{i}\right\}$ must be eventually zero; however, in order to ensure that the numbers $a_{i}$ are distinct, we need to work with the truncated version (4.3) in which the term $r_{k}$ is not necessarily 0 .

Assume now that $|m|=\left|r_{0}\right|>37$. Then

$$
a_{1}=\langle\sqrt{|m|}\rangle \geq\langle\sqrt{37}\rangle \geq 6 .
$$

Moreover, if $i \geq 2$ and $a_{i} \geq 3$ then (4.2) and (4.1) imply that

$$
3 \leq a_{i}=\left\langle\sqrt{\left|r_{i-1}\right|}\right\rangle \leq\left\langle\left|r_{i-2}\right|^{1 / 4}\right\rangle\left\langle\left\langle\sqrt{\left|r_{i-2}\right|}\right\rangle=a_{i-1},\right.
$$

since any real number $x$ with $\langle x\rangle \geq 3$ must be at least equal to $5 / 2$ and hence satisfies $x<x^{2}-1$ and $\langle x\rangle<\left\langle x^{2}\right\rangle$. Therefore, defining $k$ to be the maximal index such that $a_{k} \geq 6$, we have

$$
a_{1}>a_{2}>\ldots>a_{k} \geq 6>a_{k+1} .
$$

Furthermore, by (4.1) we have $\left\langle\sqrt{\left|r_{k}\right|}\right\rangle=a_{k+1} \leq 5$, so that $\left|r_{k}\right| \leq(5+$ $1 / 2)^{2}<36$. If $r_{k}=0$, then (4.3) gives an admissible representation of $m$. Otherwise we have $0<\left|r_{k}\right|<36$ and we can therefore apply Lemma 4.1 to represent $r_{k}$ in the form

$$
r_{k}=\sum_{i=k+1}^{t} \varepsilon_{i} a_{i}^{2}, \quad 5 \geq a_{k+1}>\ldots>a_{t} \geq 1 .
$$

Combining this representation with (4.3) we obtain again an admissible representation of $m$ involving $t \leq k+5$ squares. In either case we obtain the inequality 


$$
Q(m) \leq \sum_{i=1}^{t} a_{i} \leq \sum_{i=1}^{k} a_{i}+\sum_{i=1}^{5} i=\sum_{i=1}^{k} a_{i}+15 .
$$

To bound the sum $\sum_{i=1}^{k} a_{i}$, we first observe that by (4.2) and induction we have for each $i \geq 1$,

$$
\left|r_{i}\right| \leq\left|r_{0}\right|^{2^{-i}}=|m|^{2^{-i}} .
$$

Together with (4.1), this implies

$$
a_{i}=\left\langle\sqrt{\left|r_{i-1}\right|}\right\rangle \leq|m|^{2^{-i}}+1 / 2
$$

and, in particular,

$$
6 \leq a_{k} \leq|m|^{2^{-k}}+1 / 2 .
$$

The last estimate implies

$$
k \leq \frac{1}{\log 2} \mathcal{L}_{|m|},
$$

which in view of the inequality $t \leq k+5$ shows that the representation constructed above satisfies the additional restriction (3.9) stated in part (iv) of the theorem. Moreover, (4.5) and (4.6) yield

$$
\sum_{i=1}^{k} a_{i} \leq \sum_{i=1}^{k}\left(|m|^{2^{-i}}+1 / 2\right) \leq f(\sqrt{|m|})+O\left(\mathcal{L}_{|m|}\right) .
$$

In view of (4.4) this establishes the bound (3.7).

To prove the upper bound in (3.6), we observe that if $\sqrt{|m|}=a+\vartheta$ with $|\vartheta| \leq 1 / 2$, then we have $a=\langle\sqrt{|m|}\rangle,|\vartheta|=\theta_{|m|}$ and

$$
\left|r_{1}\right|=|| m\left|-a^{2}\right|=\left|(a+\vartheta)^{2}-a^{2}\right|=2 a|\vartheta|+O(1)=2 \theta_{|m|} \sqrt{|m|}+O(1) .
$$

Using this estimate together with (4.4), (4.5), and (4.6), we obtain

$$
\begin{aligned}
Q(m) & \leq \sum_{i=1}^{k} a_{i}+O(1) \leq \sqrt{\left|r_{0}\right|}+\sqrt{\left|r_{1}\right|}+\sum_{i=3}^{k}\left(|m|^{2^{-i}}+1 / 2\right)+O(1) \\
& =\sqrt{|m|}+\sqrt{2 \theta_{|m|}}|m|^{1 / 4}+O\left(|m|^{1 / 8}\right)
\end{aligned}
$$

which is the desired estimate.

5. Proof of Theorem 3; lower bounds. We begin with a lemma which supplies the key step in the proof.

LemmA 5.1. (i) For any integer $m$, we have $Q(m)=Q(|m|) \geq \sqrt{|m|}$.

(ii) If $m$ is a sufficiently large positive integer, then we have

$$
Q(m)=\min \left\{q+Q\left(m-q^{2}\right), q+1+Q\left(m-(q+1)^{2}\right)\right\},
$$

where $q=\lfloor\sqrt{|m|}\rfloor$. 
Proof. (i) The identity $Q(m)=Q(|m|)$ follows immediately from the definition of $Q(m)$. The bound $Q(m) \geq \sqrt{|m|}$ holds trivially for $m=0$, since $Q(0)=0$. If $m \neq 0$, then any representation of the form

$$
m=\sum_{i=1}^{t} \varepsilon_{i} a_{i}^{2}, \quad \varepsilon_{i} \in\{ \pm 1\}, a_{1}>a_{2}>\ldots>a_{t} \geq 1,
$$

satisfies

$$
\sum_{i=1}^{t} a_{i} \geq\left(\sum_{i=1}^{t} a_{i}^{2}\right)^{1 / 2} \geq\left|\sum_{i=1}^{t} \varepsilon_{i} a_{i}^{2}\right|^{1 / 2}=\sqrt{|m|} .
$$

By the definition of $Q(m)$ this implies $Q(m) \geq \sqrt{|m|}$.

(ii) We first show that $Q(m)$ is bounded from below by the right-hand side of (5.1). Suppose that $m$ is a positive integer and fix a representation of the form (5.2) such that $Q(m)=\sum_{i=1}^{t} a_{i}$. If $t=1$ in (5.2), then $|m|=$ $a_{1}^{2}=q^{2}$, and (5.1) holds trivially. Assume therefore that $t \geq 2$. By (5.2), $\sum_{i=2}^{t} \varepsilon_{i} a_{i}^{2}$ is an admissible representation for the number $m-\varepsilon_{1} a_{1}^{2}$, and we therefore have $Q\left(m-\varepsilon_{1} a_{1}^{2}\right) \leq \sum_{i=2}^{t} a_{i}$. It follows that

$$
Q(m)=a_{1}+\sum_{i=2}^{t} a_{i} \geq a_{1}+Q\left(m-\varepsilon_{1} a_{1}^{2}\right) .
$$

Thus, to obtain the lower bound in (5.1), it suffices to show that $\varepsilon_{1}=1$ and $a_{1}=q$ or $a_{1}=q+1$ whenever $m$ is sufficiently large.

Suppose first that $a_{1} \leq \sqrt{m / 2}$. Then (5.2) implies

$$
Q(m)=\sum_{i=1}^{t} a_{i} \geq \frac{1}{a_{1}} \sum_{i=1}^{t} a_{i}^{2} \geq \frac{1}{a_{1}}\left|\sum_{i=1}^{t} \varepsilon_{i} a_{i}^{2}\right|=\frac{m}{a_{1}} \geq \sqrt{2 m},
$$

which contradicts the upper bound of (3.6) if $m$ is sufficiently large. If $a_{1}>$ $\sqrt{m / 2}$ and $\varepsilon_{1}=-1$, then (5.3) and part (i) of the lemma give

$$
Q(m) \geq a_{1}+\sqrt{m+a_{1}^{2}} \geq \sqrt{m / 2}+\sqrt{3 m / 2},
$$

which again yields a contradiction to the upper bound of (3.6).

Finally, suppose that $a_{1}>\sqrt{m / 2}, \varepsilon_{1}=1$, but $a_{1} \notin\{q, q+1\}$. In this case we obtain from (5.3) and part (i) of the lemma the bound

$$
Q(m) \geq a_{1}+\sqrt{\left|m-a_{1}^{2}\right|} .
$$

Now, note that the function $x+\sqrt{\left|m-x^{2}\right|}$ is decreasing for $\sqrt{m / 2}<x$ $<\sqrt{m}$ and increasing for $x>\sqrt{m}$. Since $q \leq \sqrt{m}<q+1$, it follows that over the ranges $\sqrt{m / 2}<a_{1} \leq q-1$ and $a_{1} \geq q+2$ the right-hand side of (5.4) is minimal when $a_{1}=q-1$ or $a_{1}=q+2$, and in either case is bounded 
from below by

$$
\begin{aligned}
q-1+\min \left(\sqrt{m-(q-1)^{2}}, \sqrt{(q+2)^{2}-m}\right) \\
\quad \geq q-1+\sqrt{m-(\sqrt{m}-1)^{2}}=\sqrt{m}+\sqrt{2} m^{1 / 4}+O(1) .
\end{aligned}
$$

Since this bound exceeds the upper bound (3.6) for large enough $m$, we conclude that for sufficiently large $m, a_{1}$ must be equal to either $q$ or $q+1$, as we wanted to show.

To obtain the reverse inequality, it suffices to note that under the conditions $Q\left(m-q^{2}\right)<q$ and $Q\left(m-(q+1)^{2}\right)<q+1$ we obtain admissible representations of $m$ by adding $q^{2}$ to any admissible representation of $m-q^{2}$ or by adding $(q+1)^{2}$ to any admissible representation of $m-(q+1)^{2}$ and therefore have $Q(m) \leq \min \left(q+Q\left(m-q^{2}\right), q+1+Q\left(m-(q+1)^{2}\right)\right)$. In view of the inequalities $0 \leq m-q^{2} \leq 2 q$ and $0 \leq(q+1)^{2}-m \leq 2 q+1$ and the bound $Q(m) \ll \sqrt{|m|}$, the two conditions are satisfied provided $m$ is sufficiently large.

Remark. The recurrence formula (5.1) could be used in principle to evaluate $Q(m)$ for any $m$ to within an error term $O(1)$, but it is unlikely that it would lead to a simple explicit expression for $Q(m)$ or provide a simple algorithm for computing $Q(m)$ for any particular value of $m$ without the knowledge of the prior values of the function $Q$. The reason for this is that it seems hard to decide a priori, which of the two terms on the right of the formula achieves the minimum; in particular, since the function $Q(m)$ is not monotonic, the minimum is not necessarily attained (or even approximately attained) at the term in which the argument of $Q$ (i.e., $m-q^{2}$ or $\left.m-(q+1)^{2}\right)$ has smaller absolute value.

Proof of (3.6), lower bound. In view of part (i) of Lemma 5.1 we may assume that $m$ is sufficiently large and positive. Writing $\theta=\theta_{m}=$ $\|\sqrt{m}\|$ and $q=\lfloor\sqrt{m}\rfloor$, we have $\sqrt{m}=q+\theta$ if $q^{2} \leq m \leq q^{2}+q, \sqrt{m}=q+1-\theta$ if $q^{2}+q+1 \leq m<(q+1)^{2}$, and in any case

$$
\min \left(\left|m-q^{2}\right|,\left|m-(q+1)^{2}\right|\right) \geq 2 q \theta+O(1) .
$$

Applying Lemma 5.1, we therefore obtain

$$
\begin{aligned}
Q(m) & \geq q+\min \left(\sqrt{\left|m-q^{2}\right|}, \sqrt{\left|m-(q+1)^{2}\right|}\right) \\
& \geq q+\sqrt{2 q \theta+O(1)} \geq \sqrt{m}+\sqrt{2 \theta} m^{1 / 4}+O(1),
\end{aligned}
$$

which proves the lower bound of (3.6).

Proof of (3.8). We first note that the recurrence relation $m_{k}=$ $m_{k-1}^{2}+m_{k-1}$ implies $\left\lfloor\sqrt{m_{k}}\right\rfloor=\left\lfloor\sqrt{m_{k}+1}\right\rfloor=m_{k-1}$. Thus, if $m=m_{k}$ or $m=m_{k}+1$, then we have, in the notation of Lemma 5.1, $q=m_{k-1}$. Moreover, the numbers $m-q^{2}$ and $m-(q+1)^{2}$ are equal to $m_{k-1}$ and 
$-\left(m_{k-1}+1\right)$, respectively, if $m=m_{k}$, and to $m_{k-1}+1$ and $-m_{k-1}$ if $m=m_{k}+1$. Setting

$$
Q_{k}=\min \left(Q\left(m_{k}\right), Q\left(m_{k}+1\right)\right)
$$

and noting that $Q(m)=Q(-m)$ we therefore obtain from (5.1) the inequality

$$
Q_{k} \geq m_{k-1}+Q_{k-1}
$$

for all sufficiently large $k$, say $k \geq k_{0}$. Iterating this inequality, we deduce

$$
Q\left(m_{k}\right) \geq Q_{k} \geq \sum_{i=k_{0}-1}^{k-1} m_{i}+Q_{k_{0}-1}=\sum_{i=1}^{k-1} m_{k-i}+O(1)
$$

for $k \geq k_{0}$.

To estimate the sum on the right of (5.5), we show by induction that for $0 \leq i \leq k$

$$
m_{k-i} \leq m_{k}^{2^{-i}} \leq m_{k-i}+1-2^{-i} .
$$

For $i=0,(5.6)$ holds trivially. Assuming (5.6) holds for some $i \leq k-1$, we deduce

$$
m_{k} \geq m_{k-i}^{2^{i}} \geq\left(m_{k-i-1}^{2}\right)^{2^{i}}=m_{k-i-1}^{2^{i+1}}
$$

and

$$
\begin{aligned}
m_{k} & \leq\left(m_{k-i}+1-2^{-i}\right)^{2^{i}}=\left(m_{k-i-1}^{2}+m_{k-i-1}+1-2^{-i}\right)^{2^{i}} \\
& \leq\left(m_{k-i-1}^{2}+2\left(1-2^{-i-1}\right) m_{k-i-1}\right)^{2^{i}}<\left(m_{k-i-1}+1-2^{-i-1}\right)^{2^{i+1}},
\end{aligned}
$$

which implies (5.6) for $i+1$ and completes the induction.

Applying first (5.6) with $i=k-1$ we obtain

$$
2=m_{1} \leq m_{k}^{2^{-k+1}} \leq m_{1}+1-2^{-k+1}=3-2^{-k+1},
$$

which implies $k=\mathcal{L}_{m_{k}} / \log 2+O(1)=t_{m_{k}}+O(1)$. Using this inequality and the upper bound of (5.6) we get

$$
\begin{aligned}
\sum_{i=1}^{k} m_{k-i} & \geq \sum_{i=1}^{k}\left(m_{k}^{2^{-i}}-1\right)=\sum_{i=0}^{t_{m_{k}}}{\sqrt{m_{k}}}^{2^{-i}}-k+O(1) \\
& =f\left(\sqrt{m_{k}}\right)-\frac{1}{\log 2} \mathcal{L}_{m_{k}}+O(1),
\end{aligned}
$$

since by (5.6) the terms $m_{k}^{2^{-i}}$ with $i=k+O(1)$ are of order $O(1)$. Combined with (5.5), this gives the desired estimate.

6. Proof of Theorem 1; lower bounds. Recall that $R(s)$ is the largest integer $r$ not expressible in the form (3.4) with integers $h_{i}$ and $k_{i}$ satisfying 
(3.2) and (3.3). For $0 \leq r_{0}<4 s$ let $R\left(s, r_{0}\right)$ denote the largest such integer $r$ that lies in the residue class $r_{0}$ modulo $4 s$. Then clearly

$$
R(s)=\max _{0 \leq r_{0}<4 s} R\left(s, r_{0}\right) .
$$

We shall obtain the lower bounds of Theorem 1 by considering $R\left(s, r_{0}\right)$ for suitable choices of $r_{0}$.

We begin with a lemma which gives a bound for $R\left(s, r_{0}\right)$ in terms of the function $Q(m)$ defined in Theorem 3 .

LEMma 6.1. We have

$$
R\left(s, r_{0}\right) \geq 2 s \min \{Q(2 s-d), Q(2 s+d)\}+O(s),
$$

where $|d| \leq 2 s$ is chosen so that

$$
d \equiv \begin{cases}r_{0} \bmod 4 s & \text { if } r_{0} \text { is odd, } \\ 2 s-r_{0} \bmod 4 s & \text { if } r_{0} \text { is even. }\end{cases}
$$

Proof. It suffices to show that any integer $r \equiv r_{0} \bmod 4 s$ which has a representation of the form

$$
r=2 s \sum_{i=1}^{t}\left(h_{i}+k_{i}\right)+\sum_{i=1}^{t}\left(k_{i}^{2}-h_{i}^{2}\right)=2 s \Sigma_{1}+\Sigma_{2},
$$

say, with integers $h_{i}$ and $k_{i}$ satisfying (3.2) and (3.3), is bounded from below by the right-hand side of (6.2).

We first observe that, by the upper bound $Q(m) \leq \sqrt{|m|}+O\left(|m|^{1 / 4}\right)$ of Theorem 3, the right-hand side of (6.2) is bounded from above by

$$
2 s \min (\sqrt{2 s-d}, \sqrt{2 s+d})+O\left(s^{5 / 4}\right) \leq 2 s \sqrt{2 s}+O\left(s^{5 / 4}\right) .
$$

Thus, if

$$
r \geq 4 s^{3 / 2}+O(s)
$$

then $r$ is bounded from below by the right-hand side of (6.2).

Next, note that under the conditions $0 \leq h_{i}<s$ and $k_{i}>0$, which are implied by (3.2) and (3.3), the right-hand side of (6.4) is an increasing function of each of the variables $h_{i}$ and $k_{i}$. Hence, for any $\lambda$ with $0<\lambda \leq 1$, (6.4) implies

$$
\begin{aligned}
& r \geq 2 s \sum_{i=1}^{t}\left(\lambda h_{i}+\lambda k_{i}\right)+\sum_{i=1}^{t}\left(\left(\lambda k_{i}\right)^{2}-\left(\lambda h_{i}\right)^{2}\right) \\
& \quad \geq 2 s \lambda \Sigma_{1}+\lambda^{2} \Sigma_{2} \geq 2 s \lambda \sqrt{\left|\Sigma_{2}\right|}+\lambda^{2} \Sigma_{2},
\end{aligned}
$$

since trivially

$$
\Sigma_{1}^{2} \geq \sum_{i=1}^{t}\left(h_{i}^{2}+k_{i}^{2}\right) \geq\left|\Sigma_{2}\right| .
$$


If now $\left|\Sigma_{2}\right| \geq 4 s$, then choosing $\lambda=\sqrt{4 s /\left|\Sigma_{2}\right|}$ we obtain $r \geq 4 s^{3 / 2}-4 s$ and hence (6.5). Thus, it remains to consider the case when

$$
\left|\Sigma_{2}\right|<4 s \text {. }
$$

Observe that the sums $\Sigma_{1}$ and $\Sigma_{2}$ in (6.4) have the same parity, since $x \equiv \pm x^{2} \bmod 2$ for any integer $x$. Hence, if $r \equiv r_{0} \bmod 4 s$ with $r_{0}$ even, (6.4) implies that both sums are even and that $r_{0} \equiv \Sigma_{2} \bmod 4 s$. If $r_{0}$ is odd, both sums are odd, and in this case (6.4) yields $r_{0} \equiv \Sigma_{2}-2 s \bmod 4 s$. In either case we have $\left|\Sigma_{2}\right| \equiv 2 s+d$ or $\left|\Sigma_{2}\right| \equiv 2 s-d$ with $d$ given by (6.3). In view of (6.6), this implies

$$
\left|\Sigma_{2}\right| \in\{2 s \pm d\} .
$$

The conditions (3.2) and (3.3) imply that in the representation $\Sigma_{2}=$ $\sum_{i=1}^{t}\left(k_{i}^{2}-h_{i}^{2}\right)$ the numbers $h_{i}$ are mutually distinct and non-negative and the numbers $k_{i}$ are mutually distinct and positive, although the two sets of numbers are not necessarily disjoint. However, by dropping any pairs $\left(h_{i}, k_{j}\right)$ with $h_{i}=k_{j}$ as well as 0 if it occurs among the numbers $h_{i}$ and relabeling the remaining numbers $h_{i}$ and $k_{i}$ we obtain a representation of the form

$$
\Sigma_{2}=\sum_{i=1}^{t_{1}} k_{i}^{2}-\sum_{i=1}^{t_{2}} h_{i}^{2}
$$

in which the integers $h_{i}$ and $k_{j}$ are mutually distinct and strictly positive. The latter representation is an admissible representation in the definition of $Q\left(\Sigma_{2}\right)$, and we therefore have

$$
Q\left(\left|\Sigma_{2}\right|\right)=Q\left(\Sigma_{2}\right) \leq \sum_{i=1}^{t_{1}} k_{i}+\sum_{i=1}^{t_{2}} h_{i} \leq \Sigma_{1} .
$$

Combining this inequality with (6.7) and (6.4) yields the desired lower bound for $r$.

This completes the proof of the lemma.

Proof of (1.2), lower bound. By (6.1) and Lemma 6.1 we have

$$
R(s) \geq 2 s \max _{|d| \leq \sqrt{2 s}} \min \{Q(2 s-d), Q(2 s+d)\}+O(s) .
$$

To bound the right-hand side, we use the bound of (3.6) of Theorem 3 together with the estimates

$$
\begin{aligned}
\sqrt{2 s \pm d} & =\sqrt{2 s} \pm \frac{d}{2 \sqrt{2 s}}+O\left(s^{-1 / 2}\right) & & (|d| \leq \sqrt{2 s}), \\
(2 s \pm d)^{1 / 4} & =(2 s)^{1 / 4}+O\left(s^{-1 / 4}\right) & & (|d| \leq \sqrt{2 s}) .
\end{aligned}
$$

We thus obtain for $|d| \leq \sqrt{2 s}$,

$$
\min (Q(2 s-d), Q(2 s+d)) \geq \sqrt{2 s}+\sqrt{2 \mu}(2 s)^{1 / 4}+O\left(s^{1 / 8}\right),
$$


where

$$
\mu=\mu(s, d)=\min (\|\sqrt{2 s+d}\|,\|\sqrt{2 s-d}\|) .
$$

By (6.9) we have

$$
\|\sqrt{2 s \pm d}\|=\left\|\sqrt{2 s} \pm \frac{d}{2 \sqrt{2 s}}\right\|+O\left(s^{-1 / 2}\right)
$$

and therefore

$$
\max _{|d| \leq \sqrt{2 s}} \mu(s, d)=\max _{|\delta| \leq 1 / 2} \min \{\|\delta+\sqrt{2 s}\|,\|\delta-\sqrt{2 s}\|\}+O\left(s^{-1 / 2}\right) .
$$

It is easy to see that the maximum on $\delta$ is attained either at $\delta=0$ or at $\delta=$ $1 / 2$ and thus is equal to $\max (\|\sqrt{2 s}\|,\|\sqrt{2 s}-1 / 2\|)=\lambda_{s}^{2} / 2$ by the definition of $\lambda_{s}$. It follows that the left-hand side of (6.11) is equal to $\lambda_{s}^{2} / 2+O\left(s^{-1 / 2}\right)$, which combined with (6.10) and (6.8) proves the lower bound of (1.2).

Proof of (1.4). We set $s_{k}=m_{k} / 2$ for $k \geq 1$ with $m_{k}$ defined as in part (iii) of Theorem 3. Clearly $s_{1}=1$ and $s_{k}=2 s_{k-1}^{2}+1$ for $k \geq 2$, so that $s_{k}$ is an odd integer. Applying the bound of Lemma 6.1 with $r_{0}=2 s_{k}$ (so that $d=0$ ), together with the estimate (3.8) of Theorem 3, we obtain

$$
\begin{aligned}
R\left(s_{k}\right) & \geq R\left(s_{k}, 2 s_{k}\right) \geq 2 s_{k} Q\left(2 s_{k}\right)+O\left(s_{k}\right) \\
& \geq 2 s_{k}\left\{f\left(\sqrt{m_{k}}\right)+O\left(\mathcal{L}_{m_{k}}\right)\right\}+O\left(s_{k}\right)=2 s_{k}\left\{f\left(\sqrt{2 s_{k}}\right)+O\left(\mathcal{L}_{s_{k}}\right)\right\},
\end{aligned}
$$

which proves (1.4).

7. Proof of Theorem 1; upper bounds. To obtain the upper bounds (1.2) and (1.3) for $R(s)$, we need to show that if $r$ is greater than the right-hand side of (1.2) or (1.3) then $r$ is expressible in the form (3.4), i.e.,

$$
r=2 s \sum_{i=1}^{t}\left(h_{i}+k_{i}\right)+\sum_{i=1}^{t}\left(k_{i}^{2}-h_{i}^{2}\right),
$$

with integers $h_{i}$ and $k_{i}$ satisfying (3.2) and (3.3). In fact, it will be convenient to also consider such representations with (3.2) and (3.3) replaced by the slightly stronger conditions

$$
\begin{aligned}
& 1 \leq h_{i} \leq s-1, \quad h_{i} \text { distinct } \\
& 1 \leq k_{i} \leq s-1, \quad k_{i} \text { distinct }
\end{aligned}
$$

which have the advantage of being symmetric in $h_{i}$ and $k_{i}$. We denote by $\mathcal{R}_{t}(s)$ the set of integers $r$ expressible in the form (3.2)-(3.4), and by $\mathcal{R}_{t}^{*}(s)$ the set of integers expressible in the form (7.1)-(7.3). Needless to say, empty sums are to be interpreted as zero, so that $\mathcal{R}_{0}(s)=\mathcal{R}_{0}^{*}(s)=\{0\}$. We further set $\mathcal{R}(s)=\bigcup_{t \geq 0} \mathcal{R}_{t}(s), \mathcal{R}^{*}(s)=\bigcup_{t \geq 0} \mathcal{R}_{t}^{*}(s)$, and for any residue 
class $r_{0} \bmod 4 s$ we put

$$
\mathcal{R}_{t}\left(s, r_{0}\right)=\left\{r \in \mathcal{R}_{t}(s): r \equiv r_{0} \bmod 4 s\right\},
$$

and define $\mathcal{R}_{t}^{*}\left(s, r_{0}\right), \mathcal{R}\left(s, r_{0}\right)$, and $\mathcal{R}^{*}\left(s, r_{0}\right)$ analogously. Note that $\mathcal{R}^{*}(s) \subset$ $\mathcal{R}(s)$.

The following three propositions contain the key steps of the proof and will be proved in turn in the remainder of this section. The second and third of these propositions will be used again in Section 9 to obtain an explicit numerical bound.

Proposition 7.1. For any residue class $r_{0} \bmod 4 s$ there exists a nonnegative integer $r \in \mathcal{R}_{t}^{*}\left(s, r_{0}\right)$ for some $t \ll \mathcal{L}_{s}$ satisfying

$$
\begin{aligned}
& r \leq 2 s\left\{\sqrt{2 s}+\lambda_{s}(2 s)^{1 / 4}+O\left(s^{1 / 8}\right)\right\}, \\
& r \leq 2 s\left\{f(\sqrt{2 s})+O\left(\mathcal{L}_{s}^{2}\right)\right\} .
\end{aligned}
$$

Proposition 7.2. If $s \geq 150$ and $r \in \mathcal{R}_{t}^{*}(s)$ for some $t \leq s / 25$, then $r+4 s q \in \mathcal{R}^{*}(s)$ for every $q$ satisfying

$$
4 t+3 \leq q \leq\lfloor(s+5) / 6\rfloor s .
$$

Proposition 7.3. Suppose that $s \geq 50$ and that $\mathcal{R}^{*}(s-1)$ contains every integer in the interval $\left[(s-1)^{3} / 6,(s-1)^{3} / 2\right]$. Then $\mathcal{R}(s)$ contains every integer $\geq 2 s^{3} / 3$.

Proof of Theorem 1; upper bounds. We may clearly assume that $s$ is sufficiently large. The first two propositions imply that $\mathcal{R}^{*}(s)$, and hence also $\mathcal{R}(s)$, contains every integer $r$ in the ranges

$$
\begin{aligned}
2 s\left\{\sqrt{2 s}+\lambda_{s}(2 s)^{1 / 4}+c_{1} s^{1 / 8}\right\} & \leq r \leq 4\lfloor(s+5) / 6\rfloor s^{2}, \\
2 s\left\{f(\sqrt{2 s})+c_{2} \mathcal{L}_{s}^{2}\right\} & \leq r \leq 4\lfloor(s+5) / 6\rfloor s^{2},
\end{aligned}
$$

provided $c_{1}$ and $c_{2}$ are sufficiently large absolute constants. Since for large $s$ the ranges (7.7) and (7.8) contain the interval $\left[s^{3} / 6,2 s^{3} / 3\right]$, it follows by the third proposition that, if $s$ is sufficiently large, then $\mathcal{R}(s)$ also contains every integer $\geq 2 s^{3} / 3$. Therefore, $R(s)=\max \{r: r \notin \mathcal{R}(s)\}$ is bounded by the left-hand sides of (7.7) and (7.8), and we obtain the upper bounds of (1.2) and (1.3).

Proof of Proposition 7.1. In the case $r_{0} \equiv 0 \bmod 4 s, r=0$ belongs to $\mathcal{R}_{0}^{*}(s, 0)$ and $(7.4)$ and (7.5) are trivially satisfied. We can therefore assume that $r_{0} \not \equiv 0 \bmod 4 s$.

As a first step, we show that for sufficiently large $s$ and every integer $m$ with $0<m<4 s$ there exist integers $h_{i}$ and $k_{i}(1 \leq i \leq t)$ satisfying (7.2) and (7.3) with

$$
t \ll \mathcal{L}_{m},
$$


such that

$$
m=\sum_{i=1}^{t}\left(k_{i}^{2}-h_{i}^{2}\right)
$$

and

$$
\sum_{i=1}^{t}\left(h_{i}+k_{i}\right) \leq\left\{\begin{array}{l}
\sqrt{m}+\sqrt{2 \theta_{m}} m^{1 / 4}+O\left(m^{1 / 8}\right), \\
f(\sqrt{m})+O\left(\mathcal{L}_{m}^{2}\right)
\end{array}\right.
$$

where $\theta_{m}$ is defined as in Theorem 3 .

An application of Theorem 3 yields a representation

$$
m=\sum_{i=1}^{t_{1}} h_{i}^{2}-\sum_{i=1}^{t_{2}} k_{i}^{2}
$$

with distinct positive integers $h_{i}, 1 \leq i \leq t_{1}$, and $k_{i}, 1 \leq i \leq t_{2}$, whose sum is bounded by the right-hand side of (7.11) and such that

$$
t_{1}+t_{2} \leq C \mathcal{L}_{m}
$$

where $C$ is the constant in (3.9). The bound (7.11) implies that the integers $h_{i}$ and $k_{i}$ are bounded by $\ll \sqrt{m}<\sqrt{4 s}$, and hence are $\leq s-1$ if $s$ is sufficiently large. The conditions (7.2) and (7.3) are therefore satisfied for these integers, and if $t_{1}=t_{2}$ then (7.9)-(7.11) follow immediately with $t=t_{1}=t_{2}$.

If $t_{1} \neq t_{2}$, we will obtain (7.9)-(7.11) by suitably enlarging the sets $\left\{h_{i}\right\}$ and $\left\{k_{i}\right\}$ to two sets having the same cardinality $t$, while leaving the value of $\sum_{i} h_{i}^{2}-\sum_{i} k_{i}^{2}$ unchanged. Without loss of generality, assume that $t_{1}>t_{2}$ and set

$$
l=t_{1}-t_{2}, \quad t=t_{1}+l=t_{2}+2 l .
$$

By (7.13) we have $t \leq t_{1}+l \leq 2 t_{1} \leq 2 C \mathcal{L}_{m}$, so that (7.9) is satisfied. We define additional integers $h_{i}$ and $k_{i}$ by setting

$$
h_{t_{1}+i}=5 a_{i}, \quad k_{t_{2}+i}=3 a_{i}, \quad k_{t_{2}+l+i}=4 a_{i} \quad(1 \leq i \leq l)
$$

with distinct positive integers $a_{i}$ to be chosen later. This definition ensures that

$$
\sum_{i=1}^{t}\left(k_{i}^{2}-h_{i}^{2}\right)=\sum_{i=1}^{t_{1}} k_{i}^{2}-\sum_{i=1}^{t_{2}} h_{i}^{2},
$$

which in view of (7.12) yields (7.10). Moreover, if we restrict the integers $a_{i}$ to the residue class 1 modulo 3 , then the sets $\left\{3 a_{i}\right\},\left\{4 a_{i}\right\}$, and $\left\{5 a_{i}\right\}$ are pairwise disjoint, and the numbers defined in (7.14) are therefore mutually distinct positive integers. Thus, in order to satisfy the conditions (7.2) and (7.3), it remains to ensure that these numbers are distinct from the numbers $h_{i}, 1 \leq i \leq t_{1}$, and $k_{i}, 1 \leq i \leq t_{2}$, and are bounded by $s-1$. 
We consider the set of positive integers $a \leq 12 C \mathcal{L}_{m}+3$, where $C$ is the constant in (7.13). Clearly, at least $4 C \mathcal{L}_{m}$ of these integers satisfy the congruence $a \equiv 1 \bmod 3$, and at most $3\left(t_{1}+t_{2}\right)$ integers can be of the form $\lambda h_{i}, 1 \leq i \leq t_{1}$, or $\lambda k_{i}, 1 \leq i \leq t_{2}$, with $\lambda=1 / 3,1 / 4$, or $1 / 5$. Since by (7.13), $4 C \mathcal{L}_{m}-3\left(t_{1}+t_{2}\right) \geq t_{1}+t_{2} \geq l$, there exist $l$ of these integers, say $a_{1}, \ldots, a_{l}$, with $a_{i} \equiv 1 \bmod 3$, such that none of the integers (7.14) is equal to one of the numbers $h_{i}, 1 \leq i \leq t_{1}$, or $k_{i}, 1 \leq i \leq t_{2}$. Moreover, since $l \leq t_{1} \leq C \mathcal{L}_{m}$ and $a_{i} \leq 12 C \mathcal{L}_{m}+3$, the integers in (7.14) are bounded by $\ll \mathcal{L}_{m} \leq \mathcal{L}_{4 s}$ (and thus are $\leq s-1$ for large enough $s$ ), and we have

$$
\sum_{i=t_{1}+1}^{t} h_{i}+\sum_{i=t_{2}+1}^{t} k_{i} \ll \sum_{i=1}^{l} a_{i} \ll \mathcal{L}_{m}^{2} .
$$

Thus, extending the summation in $\sum_{i=1}^{t_{1}} h_{i}$ and $\sum_{i=1}^{t_{2}} k_{i}$ to the full range $1 \leq i \leq t$ increases the two sums by at most $O\left(\mathcal{L}_{m}^{2}\right)$, and therefore does not affect the upper bound (7.11). Hence (7.9)-(7.11) hold in any case.

Now, let $r_{0} \bmod 4 s$ be a given non-zero residue class and define $|d|<2 s$ by the congruence

$$
d \equiv \begin{cases}r_{0} \bmod 4 s & \left(r_{0} \text { odd }\right) \\ 2 s+r_{0} & \left(r_{0} \text { even }\right)\end{cases}
$$

We apply the above construction with $m=m_{ \pm}=2 s \pm d$ to obtain integers $h_{i}^{ \pm}$and $k_{i}^{ \pm}\left(1 \leq i \leq t_{ \pm}\right)$satisfying (7.2), (7.3), and (7.9)-(7.11), and set for $\varepsilon= \pm$

$$
r_{\varepsilon}=2 s \Sigma_{\varepsilon}+\varepsilon m_{\varepsilon}
$$

where $\Sigma_{\varepsilon}=\sum_{i=1}^{t_{\varepsilon}}\left(h_{i}^{\varepsilon}+k_{i}^{\varepsilon}\right)$. We shall show that at least one of the integers $r_{ \pm}$has the properties claimed in the proposition.

First note that the numbers $r_{ \pm}$are both non-negative, since $0<m_{ \pm}<4 s$ and $\Sigma_{ \pm} \geq 2$. Also, both numbers lie in the residue class $r_{0} \bmod 4 s$, since by (7.10), $\Sigma_{\varepsilon} \equiv m_{\varepsilon} \equiv d \bmod 2$ and therefore

$$
r_{\varepsilon} \equiv 2 s d+\varepsilon(2 s+\varepsilon d) \equiv 2 s(d+1)+d \equiv r_{0} \bmod 4 s .
$$

Moreover, by (7.16) and (7.10), $r_{+}$has a representation of the required form (7.1) with $t_{+} \ll \mathcal{L}_{s}$ terms, and interchanging the roles of $h_{i}^{-}$and $k_{i}^{-}$in (7.16) shows that the same is true for $r_{-}$. Therefore, we have $r_{\varepsilon} \in \mathcal{R}_{t_{\varepsilon}}^{*}\left(s, r_{0}\right)$ with $t_{\varepsilon} \ll \mathcal{L}_{s}$, and it remains to show that at least one of the integers $r_{ \pm}$is bounded by the right-hand sides of (7.4) and (7.5).

By (7.11) and (7.16) we have

$$
r_{\varepsilon} \leq\left\{\begin{array}{l}
2 s\left\{M_{\varepsilon}(d)+O\left(s^{1 / 8}\right)\right\} \\
2 s\left\{f(\sqrt{2 s+\varepsilon d})+O\left(\mathcal{L}_{s}^{2}\right)\right\}
\end{array}\right.
$$


where

$$
M_{\varepsilon}(d)=\sqrt{2 s+\varepsilon d}+\sqrt{2 \theta_{2 s+\varepsilon d}}(2 s+\varepsilon d)^{1 / 4} .
$$

The second estimate in (7.17) together with the monotonicity of the function $f(x)$ immediately gives the upper bound

$$
\min \left(r_{+}, r_{-}\right) \leq 2 s\left\{f(\sqrt{2 s})+O\left(\mathcal{L}_{s}^{2}\right)\right\},
$$

and hence the estimate (7.5) for one of the integers $r=r_{ \pm}$.

The proof of (7.4) is more involved. By (7.17) it suffices to show that for any $d$ with $|d|<2 s$,

$$
\min \left\{M_{+}(d), M_{-}(d)\right\} \leq \sqrt{2 s}+\lambda_{s}(2 s)^{1 / 4}+O\left(s^{1 / 8}\right) .
$$

To prove this estimate, we may clearly assume that $d \geq 0$. If $d>(2 s)^{3 / 4}$, then we have

$$
\sqrt{2 s-d} \leq \sqrt{2 s}-\frac{d}{2 \sqrt{2 s}} \leq \sqrt{2 s}-\frac{1}{2}(2 s)^{1 / 4},
$$

and therefore

$$
M_{-}(d) \leq \sqrt{2 s}+\frac{1}{2}(2 s)^{1 / 4},
$$

which implies (7.18) since $\lambda_{s} \geq 1 / \sqrt{2}$ for all $s$. Thus it remains to consider the case when $0 \leq d \leq(2 s)^{3 / 4}$. Setting

$$
\delta=d(2 s)^{-3 / 4}, \quad \mu=\delta(2 s)^{1 / 4}=d(2 s)^{-1 / 2},
$$

we have $0 \leq \delta \leq 1$ and hence obtain by Taylor's formula

$$
\begin{aligned}
\sqrt{2 s+\varepsilon d} & =\sqrt{2 s}+\frac{\varepsilon d}{2 \sqrt{2 s}}-\frac{d^{2}}{8(2 s)^{3 / 2}}+O\left(s^{-1 / 4}\right) \\
& =\sqrt{2 s}+\varepsilon \mu / 2-\delta^{2} / 8+O\left(s^{-1 / 4}\right)
\end{aligned}
$$

and

$$
(2 s+\varepsilon d)^{1 / 4}=(2 s)^{1 / 4}+O(1) .
$$

Thus,

$$
M_{\varepsilon}(d)=\sqrt{2 s}+(2 s)^{1 / 4}\left\{\varepsilon \delta / 2+\sqrt{2\left\|\sqrt{2 s}+\varepsilon \mu / 2-\delta^{2} / 8\right\|}\right\}+O\left(s^{1 / 8}\right),
$$

and to prove (7.18) it suffices to show that the coefficient of $(2 s)^{1 / 4}$ here is at most $\lambda_{s}$ for at least one of the choices of $\varepsilon= \pm$. This is a consequence of the following lemma.

Lemma 7.4. For $\varepsilon= \pm$ and real numbers $\theta, \delta$, and $\mu$, let

$$
\lambda_{\varepsilon}(\theta, \delta, \mu)=\varepsilon \delta / 2+\sqrt{2\left\|\theta+\varepsilon \mu / 2-\delta^{2} / 8\right\|}
$$

and put

$$
\lambda(\theta, \delta, \mu)=\min \left\{\lambda_{+}(\theta, \delta, \mu), \lambda_{-}(\theta, \delta, \mu)\right\} .
$$


Then

$$
\max _{\mu \in \mathbb{R}, \delta \geq 0} \lambda(\theta, \delta, \mu)=\sqrt{2 \max (\|\theta\|,\|\theta+1 / 2\|)} .
$$

\section{Pr o o f. Clearly}

$$
\max _{\mu \in \mathbb{R}, \delta \geq 0} \lambda(\theta, \delta, \mu) \geq \max (\lambda(\theta, 0,0), \lambda(\theta, 0,1))=\sqrt{2 \max (\|\theta\|,\|\theta+1 / 2\|)} .
$$

To obtain the inequality in the reverse direction, write

$$
\eta_{\varepsilon}=\eta_{\varepsilon}(\theta, \delta, \mu)=\sqrt{2\left\|\theta+\varepsilon \mu / 2-\delta^{2} / 8\right\|},
$$

so that

$$
\lambda(\theta, \delta, \mu)=\min \left\{\delta / 2+\eta_{+},-\delta / 2+\eta_{-}\right\} .
$$

We begin by considering $\eta_{+}^{2}+\eta_{-}^{2}$. Clearly

$$
\eta_{+}^{2}+\eta_{-}^{2}=2\left(\left\|\theta_{\delta}+\mu / 2\right\|+\left\|\theta_{\delta}-\mu / 2\right\|\right)
$$

where $\theta_{\delta}=\theta-\delta^{2} / 8$. The right-hand expression is maximal as a function of $\mu$ when the two terms $\left\|\theta_{\delta}+\mu / 2\right\|$ and $\left\|\theta_{\delta}-\mu / 2\right\|$ are equal, which is the case if and only if $\mu$ is an integer. Thus, for all real $\mu$ we have

$$
\eta_{+}^{2}(\theta, \delta, \mu)+\eta_{-}^{2}(\theta, \delta, \mu) \leq 4 \max \left(\left\|\theta_{\delta}\right\|,\left\|\theta_{\delta}-1 / 2\right\|\right)=\varrho^{2},
$$

say, where $\varrho=\varrho(\theta, \delta)$ satisfies $0 \leq \varrho \leq \sqrt{2}$. It follows that $\eta_{+} \leq \sqrt{\varrho^{2}-\eta_{-}^{2}}$, and taking the maximum over all values of $\eta_{-}$in the range $0 \leq \eta_{-} \leq \varrho$, we see that

$$
\lambda(\theta, \delta, \mu) \leq \max _{0 \leq v \leq \varrho} \min \left(\delta / 2+\sqrt{\varrho^{2}-v^{2}},-\delta / 2+v\right) .
$$

If $\delta \geq \varrho$, then for any $v$ in the interval $[0, \varrho]$ we have

$$
v-\delta / 2 \leq \varrho-\delta / 2 \leq \delta / 2 \leq \delta / 2+\sqrt{\varrho^{2}-v^{2}},
$$

so that the maximum over $v$ occurs at $v=\varrho$ and

$$
\lambda(\theta, \delta, \mu)=\varrho-\delta / 2 \leq \varrho / 2 \leq 1 / \sqrt{2} \leq \sqrt{2 \max (\|\theta\|,\|\theta+1 / 2\|)} .
$$

If $\delta<\varrho$, the maximum occurs when

$$
\delta / 2+\sqrt{\varrho^{2}-v^{2}}=-\delta / 2+v,
$$

i.e., when $v=v_{ \pm}=\delta / 2 \pm \sqrt{\left(\varrho^{2}-\delta^{2} / 2\right) / 2}$. Since $v_{-}<0<v_{+} \leq \varrho$ for $\delta<\varrho$, the maximum over $0 \leq v \leq \varrho$ is attained at $v=v_{+}$and is equal to $-\delta / 2+v_{+}=\sqrt{\left(\varrho^{2}-\delta^{2} / 2\right) / 2}$. Since

$\varrho^{2} / 2=2 \max \left(\left\|\theta-\delta^{2} / 8\right\|,\left\|\theta+1 / 2-\delta^{2} / 8\right\|\right) \leq 2 \max (\|\theta\|,\|\theta+1 / 2\|)+\delta^{2} / 4$, we obtain

$$
\lambda(\theta, \delta, \mu) \leq \sqrt{\varrho^{2} / 2-\delta^{2} / 4} \leq \sqrt{2 \max (\|\theta\|,\|\theta+1 / 2\|)} .
$$

Thus Lemma 7.4 is proved, and the proof of Proposition 7.1 is complete. 
Proof of Proposition 7.2. Let $u=\lceil q / s\rceil$ so that $(u-1) s<q \leq u s$. The bound $1 \leq q \leq\lfloor(s+5) / 6\rfloor s$ implies $1 \leq u \leq(s+5) / 6$. We shall use the identity (cf. [PS, Problem VIII.9])

$$
q=\sum_{i=1}^{u} q_{i}, \quad q_{i}=\lfloor(q+i-1) / u\rfloor .
$$

Since $q_{u}<q / u+1 \leq s+1$ and $q_{1} \geq q / u-1>(1-1 / u) s-1$, we have

$$
\left(1-\frac{1}{u}\right) s-1<q_{1} \leq q_{2} \leq \ldots \leq q_{u} \leq s .
$$

We seek to express $r+4 s q$ in the form (7.1) with $t$ replaced by $t+2 u$. For $i=1, \ldots, u$ we take

$$
h_{t+2 i-1}=k_{t+2 i-1}=m_{i}, \quad h_{t+2 i}=k_{t+2 i}=q_{i}-m_{i}
$$

for some $m_{i}$ satisfying $1 \leq m_{i} \leq q_{i}-1$ and $m_{i} \neq q_{i} / 2$. In order to ensure that the numbers $h_{1}, \ldots, h_{t+2 u}$ are distinct and the numbers $k_{1}, \ldots, k_{t+2 u}$ are distinct, we choose $m_{1}, \ldots, m_{u}$ in succession so that $1 \leq m_{i} \leq q_{i}-1$, $m_{i} \neq q_{i} / 2$, and both $m_{i}$ and $q_{i}-m_{i}$ are distinct from the $2 t+2(i-1)$ values $h_{1}, \ldots, h_{t}, k_{1}, \ldots, k_{t}, m_{1}, \ldots, m_{i-1}, q_{1}-m_{1}, \ldots, q_{i-1}-m_{i-1}$. A suitable choice of $m_{i}$ is possible, provided

$$
q_{i}-2>4 t+4(i-1) \quad(1 \leq i \leq u) .
$$

We are going to show that, under the hypotheses of the proposition, this condition is always satisfied.

Suppose first that $u=1$, i.e., $4 t+3 \leq q \leq s$. Then the assumption $q \geq 4 t+3$ guarantees that $q-2>4 t$, so that (7.19) holds.

Next, suppose that $2 \leq u<s / 15$. In view of the hypothesis $t \leq s / 25$ we then have

$$
4 t+4(i-1) \leq 4 t-4+4 u<4 s / 25+4 s / 15<4 s / 9
$$

and

$$
q_{i}-2>(1-1 / u) s-3 \geq s / 2-3>4 s / 9
$$

for $i=1, \ldots, u$, which gives again (7.19).

Finally, suppose that $s / 15 \leq u \leq(s+5) / 6$. Since by hypothesis $s \geq 150$, we have $u \geq 10$. Thus, in this case

$$
q_{i}-2>(1-1 / u) s-3 \geq 9 s / 10-3>5 s / 6
$$

and

$$
4 t+4(i-1) \leq 4 t+4 u-4<4 s / 25+4 s / 6<5 s / 6,
$$

and (7.19) follows. 
With $m_{1}, \ldots, m_{u}$ chosen as above, we have

$$
\begin{aligned}
r+4 s q & =2 s \sum_{i=1}^{t}\left(h_{i}+k_{i}\right)+\sum_{i=1}^{t}\left(k_{i}^{2}-h_{i}^{2}\right)+4 s \sum_{i=1}^{u} q_{i} \\
& =2 s \sum_{i=1}^{t+2 u}\left(h_{i}+k_{i}\right)+\sum_{i=1}^{t+2 u}\left(k_{i}^{2}-h_{i}^{2}\right),
\end{aligned}
$$

so that $r+4 s q \in \mathcal{R}^{*}(s)$ as asserted.

Proof of Proposition 7.3. Suppose $s \geq 50$ and that every integer in the interval $\left[(s-1)^{3} / 6,(s-1)^{3} / 2\right]$ belongs to $\mathcal{R}^{*}(s-1)$. Let $r \geq 2 s^{3} / 3$ be given and set $n=r+P(s)$. If $n \geq s^{5}$ then $n>N(s)$ by Theorem 2 and therefore $r>R(s)$, i.e., $r \in \mathcal{R}(s)$. We may therefore assume that $P(s)+2 s^{3} / 3 \leq n<s^{5}$. Now choose a positive integer $a$ so that $n_{1}=n-a^{2}$ satisfies

$$
P(s-1)+(s-1)^{3} / 6 \leq n_{1} \leq P(s-1)+(s-1)^{3} / 2 .
$$

This is possible, since the maximum difference between consecutive squares less than $n$ is less than $2 \sqrt{n}<2 s^{5 / 2}<(s-1)^{3} / 3$ for $s \geq 50$. Moreover, the bounds on $n$ and $n_{1}$ imply that

$$
a^{2}=n-n_{1} \geq P(s)+2 s^{3} / 3-\left(P(s-1)+(s-1)^{3} / 2\right)>s^{3} / 6>(2 s)^{2} .
$$

By (7.20) we have $n_{1}-P(s-1) \in\left[(s-1)^{3} / 6,(s-1)^{3} / 2\right]$ and hence $n_{1}-P(s-1) \in \mathcal{R}^{*}(s-1)$. By the definition of $\mathcal{R}^{*}(s-1)$ (see (3.1), (3.4), (7.2) and (7.3)) this means that $n_{1}$ is expressible as a sum of $s-1$ squares of distinct positive integers $a_{i}, i=1, \ldots, s-1$, which satisfy either $1 \leq a_{i} \leq s-1$ or $1 \leq a_{i}-(s-1) \leq s-1$ and thus in any case are bounded by $2(s-1)$. Since $a>2 s, n=n_{1}+a^{2}$ is a sum of $s$ distinct non-zero squares, i.e., $r=n-P(s)$ belongs to the set $\mathcal{R}(s)$. This proves Proposition 7.3.

8. An explicit upper bound for $N(s)$. In this section we will prove the following result, which gives an explicit upper bound for $N(s)$ for $s \geq 166$. While this bound is weaker asymptotically than the bounds of Theorem 1 , such a specific upper bound is needed in order to show that $N(s)$ is strictly increasing for $s \geq 7$ and in proving the "redundancy of coprimality" in the next section. It is also useful for computing the values of $N(s)$, as it substantially reduces the number of cases that have to be checked in determining $N(s)$.

TheOREM 4. For $s \geq 166$ we have

$$
N(s)<P(s)+2 s \sqrt{2 s}+44 s^{5 / 4}+108 s .
$$

Corollary 1. For $s \geq 166$ we have $N(s)<1.033 P(s)$. 
Proof. If $s \geq 166$, then

$$
\begin{aligned}
2 s \sqrt{2 s}+44 s^{5 / 4}+108 s & \leq s^{3}\left\{2 \sqrt{2} \cdot 166^{-3 / 2}+44 \cdot 166^{-7 / 4}+108 \cdot 166^{-2}\right\} \\
& <0.011 s^{3}<0.033 P(s) .
\end{aligned}
$$

The result now follows from Theorem 4 .

Corollary 2. If $s \geq 7$, then $N(s)<N(s+1)$.

Proof. For $s \geq 360$, Theorem 4 gives

$$
\begin{aligned}
N(s) & \leq P(s)+s^{2}\left\{2 \sqrt{2} \cdot 360^{-1 / 2}+44 \cdot 360^{-3 / 4}+108 \cdot 360^{-1}\right\} \\
& <P(s)+s^{2}<P(s+1)<N(s+1) .
\end{aligned}
$$

For the range $7 \leq s \leq 359$, the monotonicity of $N(s)$ follows from the table in Section 11 (which also shows that the inequality $N(s)<N(s+1)$ fails at $s=6)$.

To prove Theorem 4, we shall use Propositions 7.2 and 7.3, as well as the following explicit version of Proposition 7.1.

Proposition 8.1. If $s \geq 165$, then for any residue class $r_{0}$ modulo $4 s$ there exists a positive integer $r \in \mathcal{R}_{t}^{*}\left(s, r_{0}\right)$ for some $t \leq 6$ satisfying

$$
r \leq 2 s \sqrt{2 s}+44 s^{5 / 4} .
$$

Proof. We will show that for any integer $m$ with $|m| \leq 2 s$ there exist positive integers $h_{i}$ and $k_{i}(1 \leq i \leq t)$ with $t=5$ or $t=6$ satisfying (7.2) and (7.3), such that

$$
\sum_{i=1}^{t}\left(h_{i}+k_{i}\right) \leq \sqrt{2 s}+21 s^{1 / 4}
$$

and

$$
\sum_{i=1}^{t}\left(k_{i}^{2}-h_{i}^{2}\right)=m .
$$

The integer

$$
r=2 s \sum_{i=1}^{t}\left(h_{i}+k_{i}\right)+\sum_{i=1}^{t}\left(k_{i}^{2}-h_{i}^{2}\right)
$$

then belongs to $\mathcal{R}_{t}^{*}(s)$ and satisfies (8.1). Moreover, if $r_{0} \bmod 4 s$ is a given residue class, then choosing $m$ so that $m \equiv r_{0} \bmod 4 s$ if $r_{0}$ is even and $m \equiv r_{0}+2 s \bmod 4 s$ if $r_{0}$ is odd, we have $r \equiv r_{0} \bmod 4 s$ and therefore $r \in \mathcal{R}_{t}^{*}\left(s, r_{0}\right)$. Thus it remains to prove the above claim.

By interchanging the roles of $h_{i}$ and $k_{i}$, we see that it suffices to consider the case when $0 \leq m \leq 2 s$. 
Suppose first that $0 \leq m \leq 25 \sqrt{s}$. We then take $h_{1}=4$ and $h_{i}=i+4$ for $i=2,3,4,5$. The integers $h_{i}$ clearly satisfy (7.2) if $s \geq 10$. Moreover, since by Theorem 0

$$
m+\sum_{i=1}^{5} h_{i}^{2} \geq \sum_{i=1}^{5} h_{i}^{2}=246>N^{*}(5),
$$

we may choose distinct positive integers $k_{1}, \ldots, k_{5}$ such that

$$
\sum_{i=1}^{5} k_{i}^{2}=m+\sum_{i=1}^{5} h_{i}^{2}
$$

Our assumptions $m \leq 25 \sqrt{s}$ and $s \geq 165$ imply

$$
\sum_{i=1}^{5} k_{i}^{2} \leq 25 \sqrt{s}+246 \leq \sqrt{s}\left\{25+246 \cdot 165^{-1 / 2}\right\}<45 \sqrt{s}
$$

so that $k_{1}, \ldots, k_{5}$ are positive integers less than $\sqrt{45} s^{1 / 4}<s$ and therefore satisfy (7.3). Moreover,

$$
\begin{aligned}
\sum_{i=1}^{5}\left(h_{i}+k_{i}\right) & \leq 4+\sum_{i=2}^{5}(i+4)+\sqrt{5 \sum_{i=1}^{5} k_{i}^{2}} \\
& \leq 34+\sqrt{5 \cdot 45 \sqrt{s}}=34+15 s^{1 / 4},
\end{aligned}
$$

which implies the bound (8.2) with $t=5$.

Now suppose that $25 \sqrt{s}<m \leq 2 s$. In this case we take $h_{1}=1$ and $k_{1}=\lfloor\sqrt{m}\rfloor$, so that

$$
(\sqrt{m}-1)^{2}=m-2 \sqrt{m}+1<k_{1}^{2} \leq m \leq 2 s,
$$

and, in particular, $k_{1} \leq \sqrt{2 s}<s$. Setting $d=m-\left(k_{1}^{2}-h_{1}^{2}\right)=m+1-k_{1}^{2}$, we have

$$
1 \leq d<2 \sqrt{m} \leq 2 \sqrt{2 s} .
$$

The assumptions $m>25 \sqrt{s}$ and $s \geq 165$ give

$$
k_{1}^{2}>m-2 \sqrt{m}=m\left(1-\frac{2}{\sqrt{m}}\right)>25 \sqrt{s}\left(1-\frac{2}{5 \cdot 165^{1 / 4}}\right)>22 \sqrt{s} .
$$

Next, we take $h_{2}=4$ and $h_{i}=3+i$ for $3 \leq i \leq 6$, so that

$$
\sum_{i=2}^{6} h_{i}=34, \quad \sum_{i=2}^{6} h_{i}^{2}=246
$$

Applying again Theorem 0 , we obtain distinct positive integers $k_{2}, \ldots, k_{6}$ such that

$$
\sum_{i=2}^{6} k_{i}^{2}=d+\sum_{i=2}^{6} h_{i}^{2}
$$


that is

$$
\sum_{i=1}^{6} k_{i}^{2}=m+\sum_{i=1}^{6} h_{i}^{2}
$$

Clearly,

$$
\sum_{i=2}^{6} k_{i}^{2} \leq 2 \sqrt{2 s}+246 \leq \sqrt{s}\left\{2 \sqrt{2}+246 \cdot 165^{-1 / 2}\right\}<22 \sqrt{s},
$$

which by (8.4) implies that the integers $k_{2}, \ldots, k_{6}$ are less than $k_{1}$ and hence also less than $s$. The integers $h_{i}$ and $k_{i}$ therefore satisfy (7.2) and (7.3). Moreover, we have

$$
\begin{aligned}
\sum_{i=1}^{6}\left(h_{i}+k_{i}\right) & =k_{1}+\sum_{i=2}^{6} k_{i}+35 \leq k_{1}+\sqrt{5 \sum_{i=2}^{6} k_{i}^{2}}+35 \\
& \leq \sqrt{m}+\sqrt{5 \cdot 22 \sqrt{s}}+35 \leq \sqrt{2 s}+s^{1 / 4}\left\{\sqrt{110}+35 \cdot 165^{-1 / 4}\right\},
\end{aligned}
$$

which again gives (8.2).

This completes the proof of Proposition 8.1.

Proof of Theorem 4. First suppose that $s \geq 165$. By Proposition 8.1, for any residue class $r_{0}$ mod $4 s$ there exists an integer $r \leq 2 s \sqrt{2 s}+44 s^{5 / 4}$ which belongs to $\mathcal{R}_{t}^{*}\left(s, r_{0}\right)$ for some $t \leq 6$. By Proposition 7.2 , it follows that $r+4 s q \in \mathcal{R}^{*}(s)$ for any $q$ satisfying

$$
4 \cdot 6+3 \leq q \leq\lfloor(s+5) / 6\rfloor s .
$$

Since $r+4\lfloor(s+5) / 6\rfloor s^{2} \geq 2 s^{3} / 3$ and

$$
r+(4 \cdot 6+3) 4 s \leq 2 s \sqrt{2 s}+44 s^{5 / 4}+108 s
$$

we conclude that every integer in the interval $\left[2 s \sqrt{2 s}+44 s^{5 / 4}+108 s, 2 s^{3} / 3\right]$ belongs to $\mathcal{R}^{*}(s)$. Furthermore, since for $s \geq 165$,

$2 s \sqrt{2 s}+44 s^{5 / 4}+108 s \leq s^{3}\left\{2 \sqrt{2} \cdot 165^{-3 / 2}+44 \cdot 165^{-7 / 4}+108 \cdot 165^{-2}\right\}<s^{3} / 6$,

this interval contains the interval $\left[s^{3} / 6,2 s^{3} / 3\right]$ and we can apply Proposition 7.3 to deduce that for $s \geq 165+1=166, \mathcal{R}(s)$ contains every integer $\geq 2 s^{3} / 3$. Hence, for $s \geq 166$, we have

$$
R(s)=\max \{r: r \notin \mathcal{R}(s)\}<2 s \sqrt{2 s}+44 s^{5 / 4}+108 s,
$$

as claimed.

9. Redundancy of coprimality. We have stated and proved our main results in terms of the function $N(s)$, whereas Halter-Koch stated his results (as quoted in Theorem 0 ) in terms of the function $N^{*}(s)$. (Recall that $N^{*}(s)$ is defined like $N(s)$, except that only representations by sums of coprime 
squares are to be considered.) In this section we show that the two functions are identical. In fact, we prove the following more precise result.

TheOREM 5. If $s \geq 5$ and if $n$ is expressible as a sum of $s$ distinct non-zero squares, then $n$ is also expressible as a sum of $s$ distinct non-zero squares having no non-trivial common factor.

R e mark. The same result holds for $s=4$ if we restrict attention to the positive integers not divisible by 8 . It also holds for $s=3$ if we restrict attention to the positive integers not divisible by 4 (cf. [Ka], Satz 9).

Proof of Theorem 5 . For $s=5$ the assertion of the theorem follows easily from Halter-Koch's result that $N^{*}(5)=245$ (see Theorem 0). For, since $2^{2}+4^{2}+6^{2}+8^{2}+12^{2}>245$, the only integer not exceeding 245 which is expressible as a sum of 5 distinct non-zero squares having a non-trivial common factor is $220=2^{2}+4^{2}+6^{2}+8^{2}+10^{2}$, which has also the coprime representation $220=1^{2}+3^{2}+5^{2}+8^{2}+11^{2}$.

Theorem 0 also shows that $N^{*}(s)<4 P(s)$ for $6 \leq s \leq 12$. Thus every integer $\geq 4 P(s)=\sum_{i=1}^{s}(2 i)^{2}$ is expressible as a sum of $s$ distinct non-zero squares having no non-trivial common factor. If an integer less than $4 P(s)$ is expressible as a sum of $s$ distinct non-zero squares, these squares necessarily have g.c.d. 1. Indeed, otherwise the common factor of these squares would be at least $2^{2}$, and dividing each term by this factor would yield a representation of an integer less than $P(s)=\sum_{i=1}^{s} i^{2}$ as a sum of $s$ distinct squares, which is impossible. Thus the assertion of the theorem holds for $6 \leq s \leq 12$.

Now suppose that $s>12$. As before, if $n<4 P(s)$ and if $n$ is expressible as a sum of $s$ distinct non-zero squares, then these squares necessarily have g.c.d. 1 . On the other hand, if $n>3(3+\sqrt{N(s-2)+14})^{2}$, we claim that $n$ is always expressible as a sum of $s$ distinct non-zero squares having no non-trivial common factor. For if $a=\lfloor\sqrt{n / 3}+1\rfloor$ and if $n_{1}=n-a^{2}-(a+1)^{2}$, then

$$
n_{1} \geq n-(\sqrt{n / 3}+1)^{2}-(\sqrt{n / 3}+2)^{2}=(\sqrt{n / 3}-3)^{2}-14>N(s-2),
$$

and so $n_{1}$ is expressible as a sum of $s-2$ distinct non-zero squares. Since $a$ and $a+1$ are coprime and $a>\sqrt{n / 3}>\sqrt{n_{1}}$, it follows that $n$ is expressible as a sum of $s$ distinct non-zero squares having no non-trivial common factor.

To complete the proof it suffices to observe that

$$
3(3+\sqrt{N(s-2)+14})^{2}<4 P(s)
$$

for $s>12$. For $12<s \leq 168$ this follows from the computed values of $N(s)$ (see Section 11). For $s \geq 168$ we have by the first corollary to Theorem 4

$$
N(s-2)<1.033 P(s-2)<0.35(s-1)^{3},
$$


and hence

$$
\begin{aligned}
3(3+\sqrt{N(s-2)+14})^{2} & =3 N(s-2)+69+18 \sqrt{N(s-2)+14} \\
& <4 P(s-2)+4(s-1)^{2}+18 \sqrt{4(s-1)^{3} / 9} \\
& <4 P(s-2)+4(s-1)^{2}+4 s^{2}=4 P(s) .
\end{aligned}
$$

Thus the theorem is proved.

10. A result for $k$ th powers. Throughout this section, $k$ will be a fixed positive integer greater than 1 . Let $R_{s}(n)$ denote the number of solutions to $x_{1}^{k}+x_{2}^{k}+\ldots+x_{s}^{k}=n$ in non-negative integers $x_{1}, \ldots, x_{k}$. Hardy and Littlewood proved that there is a positive integer $s_{0}=s_{0}(k)$ depending only on $k$ such that if $s \geq s_{0}$ and $n$ is any positive integer, then

$$
b_{s} n^{s / k-1}<R_{s}(n)<B_{s} n^{s / k-1},
$$

where $b_{s}$ and $B_{s}$ are positive numbers depending only on $s$ and $k$ (see, e.g., Chapter 2 in $[\mathrm{Va}])$.

Lemma 10.1. There exist positive constants $C$ and $D$ (depending on $k$ ) such that if $m$ is a given positive integer and $n$ is a positive integer greater than $(C m+D)^{k}$, then $n$ is expressible as a sum of $s_{0}(k)+2$ distinct $k$-th powers each of which is $\geq m^{k}$.

Pro of. Let $F(n)$ denote the number of solutions of $x_{1}^{k}+\ldots+x_{s_{0}+2}^{k}=n$ in integers $x_{1}, \ldots, x_{s_{0}+2}$ such that $x_{i} \geq m$ for each $i$ and $x_{i} \neq x_{j}$ for $i \neq j$. Then

$$
\begin{aligned}
F(n) \geq & R_{s_{0}+2}(n)-\left(s_{0}+2\right) \sum_{i=0}^{m-1} R_{s_{0}+1}\left(n-i^{k}\right) \\
& -\left(\begin{array}{c}
s_{0}+2 \\
2
\end{array}\right) \sum_{i=m}^{\left\lfloor(n / 2)^{1 / k}\right\rfloor} R_{s_{0}}\left(n-2 i^{k}\right) \\
> & b_{s_{0}+2} n^{\left(s_{0}+2\right) / k-1}-\left(s_{0}+2\right) m B_{s_{0}+1} n^{\left(s_{0}+1\right) / k-1} \\
& -\left(\begin{array}{c}
s_{0}+2 \\
2
\end{array}\right)\left(\frac{n}{2}\right)^{1 / k} B_{s_{0}} n^{s_{0} / k-1}>0,
\end{aligned}
$$

provided

$$
b_{s_{0}+2} n^{1 / k}>\left(s_{0}+2\right) B_{s_{0}+1} m+\left(s_{0}+2\right)\left(s_{0}+1\right) 2^{-1-1 / k} B_{s_{0}} .
$$

Since the latter condition holds if $n^{1 / k}>C m+D$ with suitable constants $C$ and $D$ depending on $k$, the assertion of the lemma follows.

Let $N_{k}(s)$ denote the largest positive integer not expressible as a sum of $s$ distinct $k$ th powers of positive integers. The preceding lemma (with $m=1)$ shows that $N_{k}(s)$ exists for $s \geq s_{0}(k)+2$. 
Theorem 6. $N_{k}(s)=s^{k+1} /(k+1)+O\left(s^{k}\right)$ for $s \geq s_{0}(k)+2$.

Proof. First we note that $1+\sum_{i=1}^{s} i^{k}$ cannot be expressed as a sum of $s$ distinct positive $k$ th powers, so that

$$
N_{k}(s)>\sum_{i=1}^{s} i^{k}=\frac{s^{k+1}}{k+1}+O\left(s^{k}\right) .
$$

On the other hand, we show that if $s \geq s_{0}(k)+2$ and if

$$
n>\left(s-s_{0}-1\right)^{k+1} /(k+1)+\left(C\left(s-s_{0}-1\right)+D\right)^{k},
$$

where $C$ and $D$ are as in the lemma, then $n$ is expressible as a sum of $s$ distinct positive $k$ th powers. For then

$$
n>\sum_{i=1}^{s-s_{0}-2} i^{k}+\left(C\left(s-s_{0}-1\right)+D\right)^{k}
$$

and so by the lemma with $m=s-s_{0}-1$ we obtain that $n-\sum_{i=1}^{s-s_{0}-2} i^{k}$ is expressible as a sum of $s_{0}(k)+2$ distinct positive $k$ th powers each of which is greater than $\left(s-s_{0}-2\right)^{k}$.

11. Numerical data. We have calculated $N(s)$ for $5 \leq s \leq 400$ and for some isolated values greater than 400 . In order to carry out these computations we needed some a priori bound $L(s)$ for $N(s)$, so that it suffices to test the integers in the interval $[P(s), L(s)]$ for expressibility as a sum of $s$ distinct non-zero squares. (Recall that $P(s)=1^{2}+2^{2}+\ldots+s^{2}$ is the smallest integer expressible as a sum of $s$ distinct non-zero squares.) Given such a bound $L(s)$, we used the following algorithm: First, an array $a[P(s)], a[P(s)+1], \ldots, a[L(s)]$ is initialized to zero and a variable $L$ is set to $L(s)$. Then $s$-tuples $\left(x_{1}, \ldots, x_{s}\right)$ of integers such that $0<x_{1}<\ldots<x_{s}$ and $x_{1}^{2}+\ldots+x_{s}^{2} \leq L$ are generated, and the value of $a\left[x_{1}^{2}+\ldots+x_{s}^{2}\right]$ is set to 1 . Whenever it occurs that $x_{1}^{2}+\ldots+x_{s}^{2}=L$ exactly, then we replace $L$ by the largest integer $L^{\prime}$ such that $a\left[L^{\prime}\right]=0$. This device results in a dramatic reduction of the number of cases to be investigated compared to a brute force approach. The algorithm terminates when there is no tuple $\left(x_{1}, \ldots, x_{s}\right)$ left satisfying $0<x_{1}<\ldots<x_{s}$ and $x_{1}^{2}+\ldots+x_{s}^{2} \leq L$. The number $N(s)$ is then equal to the current value of $L$.

The efficiency of this algorithm clearly depends on the length of the array $a[n]$ and it is therefore desirable to have a numerical bound $L(s)$ which is as close to $P(s)$ as possible. For our computations of $N(s)$ for $5 \leq s \leq 400$ we used the bound given by the following proposition. While this bound is inferior asymptotically to the bound given by Theorem 4, it is better numerically for values of $s$ less than 8000 , say, as a result of the large size 
of the coefficients on the right-hand side of the inequality of Theorem 4 . The inequality of Proposition 11.1 has the disadvantage that it requires a knowledge of $N(s-1)$ and so is useful primarily for calculating a complete table of values of $N(s)$ up to some point. For calculating an isolated value of $N(s)$ we must use the bound of either Theorem 4 or Corollary 1.

Proposition 11.1. For $s \geq 6$, we have $N(s) \leq L(s)$, where

$$
L(s)=2 N(s-1)-P(s-2)+6+4 \sqrt{N(s-1)-P(s-2)+2} .
$$

Rem ark. From (1.2) it follows that

$$
\begin{aligned}
L(s)= & 2 P(s-1)-P(s-2)+2(2(s-1))^{3 / 2}+O\left(s^{5 / 4}\right) \\
& +4 \sqrt{P(s-1)-P(s-2)+O\left(s^{3 / 2}\right)} \\
= & P(s)+2(2 s)^{3 / 2}+O\left(s^{5 / 4}\right) .
\end{aligned}
$$

On the other hand, we have

$$
N(s)=P(s)+(2 s)^{3 / 2}+O\left(s^{5 / 4}\right) .
$$

Thus, $N(s)$ lies near the midpoint of the interval $[P(s), L(s)]$ for large $s$.

Proof of Proposition 11.1. Replacing $s$ by $s+1$, we see that it suffices to show that if $s \geq 5$ and

$$
n>2 N(s)-P(s-1)+6+4 \sqrt{N(s)-P(s-1)+2},
$$

then $n$ is a sum of $s+1$ distinct positive squares. We first note that (11.1) implies

$$
n>2 N(s)-P(s-1)>2 P(s)-P(s-1)>P(s-1)
$$

and so $n-P(s-1)>0$. Let $g_{s}(n)$ denote the smallest integer strictly greater than $\sqrt{(n-P(s-1)) / 2}$. To prove that $n$ is a sum of $s+1$ distinct non-zero squares it suffices to show that

$$
n-g_{s}^{2}(n)>N(s) .
$$

For if (11.2) holds, then

$$
n-g_{s}^{2}(n)=x_{1}^{2}+\ldots+x_{s}^{2},
$$

for integers $0<x_{1}<\ldots<x_{s}$ and further

$$
x_{s}^{2}=n-g_{s}^{2}(n)-x_{1}^{2}-\ldots-x_{s-1}^{2} \leq n-g_{s}^{2}(n)-P(s-1)<g_{s}^{2}(n),
$$

the last step resulting from the definition of $g_{s}(n)$. Now 


$$
g_{s}(n) \leq 1+\sqrt{(n-P(s-1)) / 2},
$$

and so to prove (11.2) it suffices to show that (11.1) implies

$$
n-\{1+\sqrt{(n-P(s-1)) / 2}\}^{2}>N(s),
$$

that is,

$$
\frac{n}{2}+\frac{P(s-1)}{2}-1-N(s)>2 \sqrt{(n-P(s-1)) / 2}
$$

or

$$
t>2 \sqrt{t-P(s-1)+1+N(s)},
$$

where

$$
t=\frac{n}{2}+\frac{P(s-1)}{2}-1-N(s) .
$$

Now (11.1) implies that certainly $t>2$. Hence (11.3) is equivalent to

$$
t^{2}>4(t-P(s-1)+1+N(s))
$$

or to

$$
t^{2}-4 t+4>4(N(s)-P(s-1)+2)
$$

or to

$$
t-2>2 \sqrt{N(s)-P(s-1)+2} .
$$

But, in view of (11.4), (11.5) is equivalent to (11.1), and hence (11.3) and (11.2) hold. This completes the proof of Proposition 11.1.

We conclude with two tables of numerical values for $N(s)$. Table I lists all values of $N(s)$ up to $s=400$ and substantially extends the table given in [HK]. Table II gives the values for $N(s)$ for $s=20,40, \ldots, 1000$, along with the polynomial approximation $P(s)$, the difference $R(s)=N(s)-P(s)$, and the approximation $R_{0}(s)=2 s\left(\sqrt{2 s}+\lambda_{s}(2 s)^{1 / 4}\right)$ to $R(s)$ given by formula (1.2) of Theorem 1. It is apparent from this table that $P(s)$ is very close to $N(s)$, the difference $R(s)=N(s)-P(s)$ being roughly of size $\sqrt{N(s)}$. On the other hand, the agreement between $R(s)$ and $R_{0}(s)$ is rather poor. The ratio $R(s) / R_{0}(s)$ between the two quantities, which by (1.2) is asymptotically equal to 1 , falls roughly between 1.15 and 1.4 for the computed values with $180 \leq s \leq 1000$. This, however, is not surprising, since the error term in (1.2) is only by a factor $O\left(s^{-1 / 8}\right)$ smaller than the main term. In fact, a careful analysis of the proof reveals that this error term oscillates in a manner similar to the term $\lambda_{s}(2 s)^{1 / 4}$, with amplitudes of size $O\left(s^{-1 / 8}\right)$ relative to the main term. 
Table I. Values of $N(s)$ for $5 \leq s \leq 400$

\begin{tabular}{|c|c|c|c|c|c|c|c|c|}
\hline$s$ & $N(s)$ & $N(s+50)$ & $N(s+100)$ & $N(s+150)$ & $N(s+200)$ & $N(s+250)$ & $N(s+300)$ & $N(s+350)$ \\
\hline & & 47668 & & & 2739627 & $521076 ?$ & 9157781 & 14503694 \\
\hline 2 & & & & & & & & 010 \\
\hline 3 & & 53097 & & & 1768 & 47969 & 342342 & 4752167 \\
\hline 4 & & 56253 & 385598 & & 864188 & & 9433610 & 4877763 \\
\hline 5 & 245 & 59522 & 396675 & 1261760 & 06277 & 5577097 & 9527815 & 15003868 \\
\hline 6 & 333 & 02 & & & & & 543 & 0352 \\
\hline 7 & 330 & & & & & & & 258213 \\
\hline 8 & 462 & & & & & & & 6457 \\
\hline 9 & 539 & & & & & & & 15516778 \\
\hline 10 & 647 & 356 & & & & $\$ 76$ & 541 & 462 \\
\hline 1 & 888 & & & & & & & 703 \\
\hline 12 & 1036 & & & & & & 742 & 827 \\
\hline 3 & 1177 & & & & & & & 8472 \\
\hline 4 & 1445 & 211 & & & & 750 & 475 & 428 \\
\hline 5 & 1722 & & & & & & & 557 \\
\hline 16 & 1990 & & & & & & & \\
\hline 7 & 2311 & & & & & & & \\
\hline 8 & 2672 & & & & & & & 866 \\
\hline 9 & 3047 & & & & & & & 303 \\
\hline 20 & 3492 & & & & & & & 287 \\
\hline 21 & 4093 & & & & & & & \\
\hline 2 & 4613 & & & & & & & 940 \\
\hline 23 & 5138 & & & & & & & 277 \\
\hline 4 & & & & & & & & 653 \\
\hline 25 & 6379 & & & & & & & 362 \\
\hline 6 & 7123 & & & & & & & \\
\hline 7 & & & & & & & & \\
\hline 8 & & & & & & & & 567 \\
\hline 29 & 9537 & & & & & & & 728 \\
\hline 30 & 10393 & & & & & & & \\
\hline 31 & 558 & & & & & & & 38009 \\
\hline 32 & & & & & & & & 3837 \\
\hline 33 & 7443 & & & & & & & 2238 \\
\hline 34 & 1863 & & & & & & & 8978927 \\
\hline 35 & $\$ 252$ & & & & & & & 9128087 \\
\hline 36 & & & & & & & & 77167 \\
\hline 37 & 8957 & & & & & & & 9426163 \\
\hline 38 & 0481 & & & & & & & \\
\hline 39 & 2042 & & & & 96062 & 8108466 & 13069884 & 19728777 \\
\hline 40 & 3678 & & & & & & & 30957 \\
\hline 41 & 6347 & & & & & & & 0032714 \\
\hline 42 & 7207 & & & & & & & 20187950 \\
\hline 43 & 9092 & & & & & & & 20342319 \\
\hline 44 & & & & & & & 13654110 & 20497803 \\
\hline & & & & & & 8622718 & 13774483 & 20653912 \\
\hline
\end{tabular}


Table I (cont.)

\begin{tabular}{|c|c|c|c|c|c|c|c|c|}
\hline$s$ & $N(s)$ & $N(s+50)$ & $N(s+100)$ & $N(s+150)$ & $N(s+200)$ & $N(s+250)$ & $N(s+300)$ & $N(s+350)$ \\
\hline 46 & 35289 & 304338 & 1056663 & 2541621 & 5009368 & 8710678 & 13894279 & 20810636 \\
47 & 37653 & 313747 & 1078332 & 2579933 & 5070898 & 8798963 & 14014592 & 20968325 \\
48 & 40042 & 323272 & 1099592 & 2619433 & 5132474 & 8887116 & 14135652 & 21126114 \\
49 & 42487 & 333123 & 1122933 & 2659421 & 5194122 & 8977320 & 14256960 & 21284758 \\
50 & 45023 & 343296 & 1145117 & 2699146 & 5256211 & 9067396 & 14379001 & 21446362 \\
\hline
\end{tabular}

Table II. Comparison between $R(s)=N(s)-P(s)$ and $R_{0}(s)=2 s\left(\sqrt{2 s}+\lambda_{s}(2 s)^{1 / 4}\right)$

\begin{tabular}{|r|r|r|r|r|c|}
\hline$s$ & $N(s)$ & $P(s)$ & $R(s)$ & $R_{0}(s)$ & $R(s) / R_{0}(s)$ \\
\hline 20 & 3492 & 2870 & 622 & 334.029 & 1.86212 \\
40 & 23678 & 22140 & 1538 & 941.071 & 1.63431 \\
60 & 76356 & 73810 & 2546 & 1693.18 & 1.50368 \\
80 & 177622 & 173880 & 3742 & 2500.56 & 1.49646 \\
100 & 343296 & 338350 & 4946 & 3464.73 & 1.42753 \\
120 & 589270 & 583220 & 6050 & 4655.05 & 1.29966 \\
140 & 932732 & 924490 & 8242 & 5521.97 & 1.49258 \\
160 & 1387602 & 1378160 & 9442 & 6917.42 & 1.36496 \\
180 & 1971412 & 1960230 & 11182 & 8356.78 & 1.33807 \\
200 & 2699146 & 2686700 & 12446 & 9788.85 & 1.27145 \\
220 & 3588112 & 3573570 & 14542 & 11196.1 & 1.29884 \\
240 & 4652746 & 4636840 & 15906 & 12548.1 & 1.26761 \\
260 & 5910676 & 5892510 & 18166 & 13792.5 & 1.31709 \\
280 & 7376258 & 7356580 & 19678 & 15484.1 & 1.27085 \\
300 & 9067396 & 9045050 & 22346 & 17651.3 & 1.26597 \\
320 & 11000162 & 10973920 & 26242 & 18676.9 & 1.40505 \\
340 & 13185828 & 13159190 & 26638 & 20926.8 & 1.27291 \\
360 & 15646462 & 15616860 & 29602 & 22362.5 & 1.32373 \\
380 & 18394212 & 18362930 & 31282 & 24660.5 & 1.26851 \\
400 & 21446362 & 21413400 & 32962 & 25835.5 & 1.27584 \\
420 & 24820272 & 24784270 & 36002 & 28789 & 1.25055 \\
440 & 28527862 & 28491540 & 36322 & 30029.4 & 1.20955 \\
460 & 32590736 & 32551210 & 39526 & 32030.6 & 1.23401 \\
480 & 37020622 & 36979280 & 41342 & 35001.3 & 1.18116 \\
500 & 41836864 & 41791750 & 45114 & 36507.2 & 1.23576 \\
520 & 47051574 & 47004620 & 46954 & 37723.2 & 1.24470 \\
540 & 52686788 & 52633890 & 52898 & 40770.3 & 1.29746 \\
560 & 58748358 & 58695560 & 52798 & 43740.1 & 1.20708 \\
580 & 65260300 & 65205630 & 54670 & 45867.6 & 1.19191 \\
600 & 72238998 & 72180100 & 58898 & 47553.7 & 1.23856 \\
620 & 79695548 & 79634970 & 60578 & 49233.6 & 1.23042 \\
640 & 87651558 & 87586240 & 65318 & 51494.2 & 1.26845 \\
660 & 96114808 & 96049910 & 64898 & 54439.4 & 1.19211 \\
\hline & & & & & \\
& & & &
\end{tabular}


Table II (cont.)

\begin{tabular}{|c|c|c|r|r|c|}
\hline$s$ & $N(s)$ & $P(s)$ & $R(s)$ & $R_{0}(s)$ & $R(s) / R_{0}(s)$ \\
\hline 680 & 105111458 & 105041980 & 69478 & 57337 & 1.21175 \\
700 & 114650008 & 114578450 & 71558 & 60199.9 & 1.18867 \\
720 & 124748958 & 124675320 & 73638 & 63034.6 & 1.16822 \\
740 & 135427128 & 135348590 & 78538 & 65844 & 1.19279 \\
760 & 146694958 & 146614260 & 80698 & 68628.9 & 1.17586 \\
780 & 158574168 & 158488330 & 85838 & 71388 & 1.20241 \\
800 & 171074942 & 170986800 & 88142 & 74119.3 & 1.18919 \\
820 & 184215972 & 184125670 & 90302 & 76819.2 & 1.17551 \\
840 & 198016654 & 197920940 & 95714 & 79483.2 & 1.20420 \\
860 & 212483472 & 212388610 & 94862 & 82105.5 & 1.15537 \\
880 & 227644810 & 227544680 & 100130 & 84679 & 1.18247 \\
900 & 243507824 & 243405150 & 102674 & 87194.8 & 1.17752 \\
920 & 260091014 & 259986020 & 104994 & 89641.3 & 1.17127 \\
940 & 277414216 & 277303290 & 110926 & 92004 & 1.20567 \\
960 & 295486262 & 295372960 & 113302 & 94262.8 & 1.20198 \\
980 & 314326668 & 314211030 & 115638 & 96389.7 & 1.19969 \\
1000 & 333951594 & 333833500 & 118094 & 99427.2 & 1.18774 \\
\hline
\end{tabular}

\section{References}

[HK] F. Halter-Koch, Darstellung natürlicher Zahlen als Summe von Quadraten, Acta Arith. 42 (1982), 11-20

[Ka] M. Kassner, Darstellungen mit Nebenbedingungen durch quadratische Formen, J. Reine Angew. Math. 331 (1982), 151-161.

[PS] G. Pólya and G. Szegö, Problems and Theorems in Analysis, Vol. II, Springer, Berlin, 1978.

[Va] R. C. Vaughan, The Hardy-Littlewood Method, Cambridge University Press, Cambridge, 1981.

[Wr] E. M. Wright, The representation of a number as a sum of five or more squares, Quart. J. Math. Oxford Ser. 4 (1933), 37-51.

DEPARTMENT OF MATHEMATICS UNIVERSITY OF ILLINOIS URBANA, ILLINOIS 61801 U.S.A.
DEPARTMENT OF COMPUTER SCIENCE UNIVERSITY OF CINCINNATI CINCINNATI, OHIO 45221 U.S.A. 